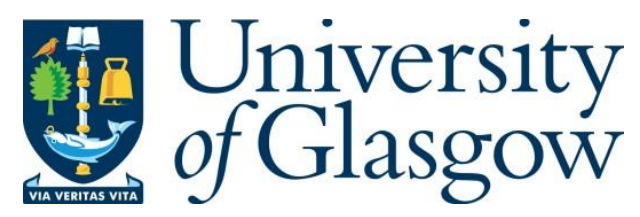

Bartel, A. and Lenstra, H. W. (2017) Commensurability of automorphism groups. Compositio Mathematica, 153(2), pp. 323-346.

There may be differences between this version and the published version. You are advised to consult the publisher's version if you wish to cite from it.

http://eprints.gla.ac.uk/149645/

Deposited on: 11 October 2017

Enlighten - Research publications by members of the University of Glasgow http://eprints.gla.ac.uk 


\title{
COMMENSURABILITY OF AUTOMORPHISM GROUPS
}

\author{
ALEX BARTEL AND HENDRIK W. LENSTRA JR.
}

\begin{abstract}
We develop a theory of commensurability of groups, of rings, and of modules. It allows us, in certain cases, to compare sizes of automorphism groups of modules, even when those are infinite. This work is motivated by the Cohen-Lenstra heuristics on class groups.
\end{abstract}

\section{INTRODUCTION}

Often, when a mathematical object is drawn in some "random" manner, the probability that it is isomorphic to a given object is inversely proportional to the size of the automorphism group of the latter. The Cohen-Lenstra heuristics [3, 4], which make predictions on the distribution of class groups of "random" algebraic number fields, are, as we intend to show, a special case of this rule, provided that one passes to Arakelov class groups. Now, Arakelov class groups may have infinitely many automorphisms, so a difficulty arises in comparing the sizes of their automorphism groups. This difficulty is resolved in the present paper. We will address the number-theoretic implications in a later one.

Our main result, formulated as Theorem 1.2 below, expresses that, for certain pairs of modules $L$ and $M$ over certain types of ring, one can meaningfully define the ratio of the size of the automorphism group Aut $M$ of $M$ to the size of Aut $L$, even when their orders \# Aut $M$ and \# Aut $L$ are infinite. If Aut $L$ can be naturally embedded in Aut $M$ as a subgroup of finite index, then the ratio mentioned may be defined to be that index. Our approach consists of giving a canonical definition of an "index of automorphism groups", to be denoted by ia $(L, M)$, in a more general situation.

As a concrete example, we consider modules over group rings. Denote by $\mathbb{Z}$ the ring of integers, by $\mathbb{Q}$ the field of rational numbers, by $\mathbb{Q}>0$ the multiplicative group of positive rational numbers, by $R[G]$ the group ring of a group $G$ over a ring $R$, and by $(G: H)$ the index of a subgroup $H$ of a group $G$. By "module" we shall always mean "left module".

Theorem 1.1. Let $G$ be a finite group, let $V$ be a finitely generated $\mathbb{Q}[G]$ module, and put $\mathcal{S}=\left\{L: L\right.$ is a finitely generated $\mathbb{Z}[G]$-module with $\mathbb{Q} \otimes_{\mathbb{Z}}$ $L \cong V$ as $\mathbb{Q}[G]$-modules $\}$. Then there exists a unique function ia: $\mathcal{S} \times \mathcal{S} \rightarrow$ $\mathbb{Q}>0$ such that

Date: October 4, 2016.

2010 Mathematics Subject Classification. 11R29, 16H20, 16K20, 18 E35.

Keywords. Calculus of fractions, Cohen-Lenstra heuristics, commensurability, ring isogenies, semisimple rings.

The first author gratefully acknowledges the financial support of the Royal Commission for the Exhibition of 1851. 
(a) if $L, L^{\prime}, M, M^{\prime} \in \mathcal{S}$ and $L \cong L^{\prime}, M \cong M^{\prime}$, then $\mathrm{ia}(L, M)=\mathrm{ia}\left(L^{\prime}, M^{\prime}\right)$;

(b) if $L, M, N \in \mathcal{S}$, then ia $(L, M) \cdot \mathrm{ia}(M, N)=\mathrm{ia}(L, N)$;

(c) if $M \in \mathcal{S}$, and $L \subset M$ is a submodule of finite index, then with $H=$ $\{\sigma \in$ Aut $M: \sigma L=L\}$ and $\rho: H \rightarrow$ Aut $L$ mapping $\sigma \in H$ to $\sigma \mid L$, one has

$$
\operatorname{ia}(L, M)=\frac{(\text { Aut } M: H) \cdot \# \operatorname{ker} \rho}{(\text { Aut } L: \rho H)} .
$$

To explain part (c), we remark that it is not hard to show that one has $L \in \mathcal{S}$, and that the three cardinal numbers (Aut $M: H)$, \# ker $\rho$, (Aut $L: \rho H$ ) are finite (see Section 7). Since these three numbers may be thought of as the ratio of the sizes of Aut $M$ and $H$, of $H$ and $\rho H$, and of Aut $L$ and $\rho H$, respectively, one may think of the expression in (c) as the ratio of the sizes of Aut $M$ and Aut $L$. The same argument shows that one has indeed ia $(L, M)=(\#$ Aut $M) / \#$ Aut $L$ if Aut $M$ and Aut $L$ are finite.

As an example, let $G$ be the trivial group, and put $n=\operatorname{dim}_{\mathbb{Q}} V$. Then each $L \in \mathcal{S}$ is isomorphic to the direct sum of $\mathbb{Z}^{n}$ with a finite abelian group $L_{0}$, and Aut $L$ is isomorphic to a semidirect product $\operatorname{Hom}\left(\mathbb{Z}^{n}, L_{0}\right) \rtimes\left(\right.$ Aut $L_{0} \times$ $\operatorname{GL}(n, \mathbb{Z}))$, where both $\operatorname{Hom}\left(\mathbb{Z}^{n}, L_{0}\right)$ and Aut $L_{0}$ are finite. Writing $M \in \mathcal{S}$ similarly, and "cancelling" $\mathrm{GL}(n, \mathbb{Z})$, one is led to believe that

$$
\operatorname{ia}(L, M)=\frac{\# \operatorname{Hom}\left(\mathbb{Z}^{n}, M_{0}\right) \cdot \# \operatorname{Aut} M_{0}}{\# \operatorname{Hom}\left(\mathbb{Z}^{n}, L_{0}\right) \cdot \# \operatorname{Aut} L_{0}}=\frac{\left(\# M_{0}\right)^{n} \cdot \# \text { Aut } M_{0}}{\left(\# L_{0}\right)^{n} \cdot \# \operatorname{Aut} L_{0}} .
$$

Making this informal argument rigorous (see Proposition 8.4), one discovers that if a function as in Theorem 1.1 exists, it must be given by the formula just stated. However, that this formula does define a function meeting all conditions, in particular (c), is not obvious. Likewise, for general $G$ the uniqueness statement of Theorem 1.1 is easy by comparison to the existence statement. Our proof of Theorem 1.1 is given in Section 8 .

There is little doubt that one can prove Theorem 1.1 using a suitable theory of covolumes of arithmetic groups. Instead, we will give an entirely algebraic proof, obtaining the theorem as a special case of a much more general result, of which the formulation requires some terminological preparation.

Isogenies. A group isogeny is a group homomorphism $f: H \rightarrow G$ with \# ker $f<\infty$ and $(G: f H)<\infty$, and its index $\mathrm{i}(f)$ is defined to be $(G: f H) / \#$ ker $f$. For a ring $R$, an $R$-module isogeny is an $R$-module homomorphism that is an isogeny as a map of additive groups. A ring isogeny is a ring homomorphism that is an isogeny as a map of additive groups. The index of an isogeny of one of the latter two types is defined as the index of the induced group isogeny on the additive groups.

Commensurabilities. If $X, Y$ are objects of a category $\mathcal{C}$, then a correspondence from $X$ to $Y$ in $\mathcal{C}$ is a triple $c=(W, f, g)$, where $W$ is an object of $\mathcal{C}$ and $f: W \rightarrow X$ and $g: W \rightarrow Y$ are morphisms in $\mathcal{C}$; we will often write $c: X \rightleftharpoons Y$ to indicate a correspondence. A group commensurability is a correspondence $c=(W, f, g)$ in the category of groups for which both $f$ and $g$ are isogenies, and the index $\mathrm{i}(c)$ of such an isogeny is defined to be $\mathrm{i}(g) / \mathrm{i}(f)$. For a ring $R$, one defines $R$-module commensurabilities and their indices analogously, replacing the category of groups by the category of $R$-modules. Likewise, one defines ring commensurabilities and their indices. 
Endomorphisms and automorphisms. Let $R$ be a ring, and let $c=(N, f, g)$ : $L \rightleftharpoons M$ be a correspondence of $R$-modules. We define the endomorphism ring End $c$ of $c$ to be the subring $\{(\lambda, \nu, \mu) \in($ End $L) \times($ End $N) \times($ End $M)$ : $\lambda f=f \nu, \mu g=g \nu\}$ of the product ring $($ End $L) \times($ End $N) \times($ End $M)$. There are natural ring homomorphisms End $c \rightarrow$ End $L$ and End $c \rightarrow$ End $M$ sending $(\lambda, \nu, \mu)$ to $\lambda$ and $\mu$, respectively; we shall write $\mathrm{e}(c)$ : End $L \rightleftharpoons$ End $M$ for the ring correspondence consisting of End $c$ and those two ring homomorphisms. Similarly, writing $E^{\times}$for the multiplicative group of invertible elements of a ring $E$, we define the automorphism group Aut $c$ of $c$ to be the group $(\text { End } c)^{\times}$, and we write a $(c):$ Aut $L \rightleftharpoons$ Aut $M$ for the group correspondence consisting of Aut $c$ and the natural maps Aut $c \rightarrow$ Aut $L$, Aut $c \rightarrow$ Aut $M$.

A domain is a non-zero commutative ring in which the product of any two non-zero elements is non-zero. A ring is semisimple if all short exact sequences of modules over the ring split.

We can now formulate the general result that we announced.

Theorem 1.2. Let $Z$ be an infinite domain such that for all non-zero $m \in Z$ the ring $Z / m Z$ is finite, let $Q$ be the field of fractions of $Z$, let $A$ be a semisimple $Q$-algebra of finite vector space dimension over $Q$, let $R \subset A$ be a sub-Z-algebra with $Q \cdot R=A$, and let $L, M$ be finitely generated $R$-modules. Then:

(a) there is an $R$-module commensurability $L \rightleftharpoons M$ if and only if the $A$ modules $Q \otimes_{Z} L$ and $Q \otimes_{Z} M$ are isomorphic;

(b) if $c$ : $L \rightleftharpoons M$ is an $R$-module commensurability, then $\mathrm{e}(c)$ : End $L \rightleftharpoons$ End $M$ is a ring commensurability, and a $(c)$ : Aut $L \rightleftharpoons$ Aut $M$ is a group commensurability;

(c) if $c, c^{\prime}: L \rightleftharpoons M$ are $R$-module commensurabilities, then one has

$$
\mathrm{i}(\mathrm{e}(c))=\mathrm{i}\left(\mathrm{e}\left(c^{\prime}\right)\right), \quad \mathrm{i}(\mathrm{a}(c))=\mathrm{i}\left(\mathrm{a}\left(c^{\prime}\right)\right) .
$$

The proof of Theorem 1.2 is given in Section 8. The essential statement is part (c).

The theorem shows that one can define ia $(L, M)=\mathrm{i}(\mathrm{a}(c))$, independently of $c$, if one has $Q \otimes_{Z} L \cong_{A} Q \otimes_{Z} M$ and $c: L \rightleftharpoons M$ is an $R$-module commensurability. One deduces the existence part of Theorem 1.1 from Theorem 1.2 by putting $Z=\mathbb{Z}, Q=\mathbb{Q}, A=\mathbb{Q}[G]$, and $R=\mathbb{Z}[G]$. Other cases that may arise in applications include localisations and completions of $\mathbb{Z}$ in the rôle of $Z$, and quotients of $Z[G]$ in the rôle of $R$.

Isogenies, commensurabilities, and their indices have many formal properties, and it is to these that Section 2 is devoted. Among other things, we define a notion of equivalence of correspondences and, under certain conditions, the composition $d \circ c$ of two correspondences $d$ and $c$. The index of a commensurability depends only on its equivalence class, and it is multiplicative in composition of commensurabilities. We introduce, for each object $L$ in the category under discussion, a group $G_{L}$ of which the elements are the equivalence classes of commensurabilities $L \rightleftharpoons L$. The group $G_{L}$ plays an important rôle in the paper. It may be thought of as the automorphism group of $L$ in a "category of fractions" [7], which is obtained by formally inverting 
all isogenies in our category. We also recall in Section 2 an explicit construction of that category of fractions: the morphisms are equivalence classes of skew correspondences, which are correspondences $(W, f, g)$ in which $f$ is an isogeny.

Section 3, on ring isogenies, culminates in the following result, which is proved as Theorem 3.8. We shall use it to pass from endomorphism rings of module commensurabilities to automorphism groups.

Theorem 1.3. Let $E \rightarrow F$ be a ring isogeny. Then the induced group homomorphism $E^{\times} \rightarrow F^{\times}$is a group isogeny. If in addition the map $E \rightarrow F$ is surjective, then so is the induced map $E^{\times} \rightarrow F^{\times}$.

In Section 4 we prove a property of the rings $R$ appearing in Theorem 1.2 that allows us to apply the results of Section 2 to the category of finitely generated $R$-modules.

Theorem 1.4. Let $R$ be a ring as in the statement of Theorem 1.2. Then $R$ is left-noetherian and right-noetherian.

For a proof, see Theorem 4.2. The point of Theorem 1.4 is that $R$ is not required to be finitely generated as a $Z$-module. As an aside, we characterise, in Theorem 4.5, the rings $Z$ satisfying the hypotheses of Theorem 1.2.

Section 5 furnishes the deus ex machina of the paper.

Theorem 1.5. Let $B$ be a semisimple ring that is finitely generated as a module over its centre $\mathrm{Z}(B)$. Then $B^{\times} /\left(\mathrm{Z}(B)^{\times}\left[B^{\times}, B^{\times}\right]\right)$is an abelian group of finite exponent.

This is proved as Theorem 5.6. In fact, we prove an explicit version of Theorem 1.5. A central simple algebra over a field $k$ is a $\operatorname{ring} B$ that is simple in the sense that it has precisely two two-sided ideals; that has centre equal to $k$; and that has finite dimension as a vector space over $k$; it is a well-known result $[6,(7.22)]$ that, under these conditions, that dimension is a square.

Theorem 1.6. Let $k$ be a field, and let $B$ be a central simple algebra over $k$. Let the dimension of $B$ as a vector space over $k$ be $d^{2}$, where $d$ is a positive integer. Then the group $B^{\times} /\left(k^{\times}\left[B^{\times}, B^{\times}\right]\right)$is abelian of exponent dividing $d$.

Our proof of Theorem 1.6 (see Theorem 5.5) makes use of Wedderburn's factorisation theorem for polynomials over division rings. Theorem 1.5 is an immediate consequence of Theorem 1.6.

In Section 6 we place ourselves in the situation of Theorem 1.2, but replacing the semisimplicity assumption on $A$ by the condition that $R$ be left-noetherian; by Theorem 1.4 this is a weaker condition. We apply the construction of Section 2 to the category of finitely generated $R$-modules, and obtain a "category of fractions" with the same objects, but with morphisms given by equivalence classes of skew correspondences. Elaborating upon a well-known argument that is ascribed to Serre, we prove that there is an equivalence of the latter category with the category of finitely generated $A$-modules that sends an $R$-module $L$ to the $A$-module $Q \otimes_{Z} L$. This has two important consequences. The first is part (a) of Theorem 1.2, which is contained in Theorem 6.3. The second is that, for a finitely generated 
$R$-module $L$, the group $G_{L}$ introduced in Section 2 may be identified with the group $\operatorname{Aut}_{A}\left(Q \otimes_{Z} L\right)$.

Section 7 uses the same hypotheses on $A$ and $R$ as Section 6 . It starts off with the proof that, for any commensurability $c: L \rightleftharpoons M$ of finitely generated $R$-modules, the correspondence $\mathrm{e}(c)$ : End $L \rightleftharpoons$ End $M$ is a ring commensurability; by Theorem 1.3, one then also obtains a group commensurability a $(c)$ : Aut $L \rightleftharpoons$ Aut $M$. This proves part (b) of Theorem 1.2. Next, we prove in Theorem 7.3 that, for commensurabilities $c: L \rightleftharpoons M$ and $d: M \rightleftharpoons N$ of finitely generated $R$-modules, one has

$$
\mathrm{i}(\mathrm{e}(d \circ c))=\mathrm{i}(\mathrm{e}(d)) \mathrm{i}(\mathrm{e}(c)), \quad \mathrm{i}(\mathrm{a}(d \circ c))=\mathrm{i}(\mathrm{a}(d)) \mathrm{i}(\mathrm{a}(c)) .
$$

This result at once allows us to reduce the proof of Theorem 1.2(c) to the special case that $L=M$, and shows that $\mathrm{i} \circ \mathrm{e}$ and $\mathrm{i} \circ$ a give rise to group homomorphisms $G_{L} \rightarrow \mathbb{Q}_{>0}$; the statement of Theorem 1.2(c) is equivalent to these homomorphisms being trivial. If we write $B=\operatorname{End}_{A}\left(Q \otimes_{Z} L\right)$, then Section 6 enables us to identify $G_{L}$ with $B^{\times}=\operatorname{Aut}_{A}\left(Q \otimes_{Z} L\right)$ and to prove that the homomorphisms are trivial on the subgroup $\mathrm{Z}(B)^{\times}$of $B^{\times}$.

In Section 8, the assumption that $A$ be semisimple is brought back in. It implies that the ring $B$ just defined is also semisimple. Since the group homomorphisms ioe and ioa are not only trivial on $\mathrm{Z}(B)^{\times} \subset B^{\times}$, but also on the commutator subgroup $\left[B^{\times}, B^{\times}\right]$, Theorem 1.2(c) becomes an immediate consequence of Theorem 1.5. We give an example to show that, unlike parts (a) and (b), part (c) of Theorem 1.2 may fail if $R$ is left-noetherian, but $A$ is not semisimple. In the same section, we prove Theorem 1.1 by putting $R=$ $\mathbb{Z}[G]$; as far as we are aware, this special case of Theorem 1.2 is essentially as hard as the general case.

Acknowledgements. We would like to thank the referees for helpful comments.

\section{ISOGENIES AND COMMENSURABILITIES}

This section is devoted to formal properties of isogenies and commensurabilities, and of their indices.

We begin by recalling a basic notion from category theory, for which we refer to [9, Ch. I, §11]. Let $L \stackrel{f}{\rightarrow} M \stackrel{g}{\leftarrow} N$ be a diagram in a category $\mathcal{C}$. We say that $\left(L \times_{M} N, p_{0}, p_{1}\right)$ is a fibre product of $L$ and $N$ over $M$ if $L \stackrel{p_{0}}{\leftarrow} L \times_{M} N \stackrel{p_{1}}{\rightarrow} N$ is a diagram in $\mathcal{C}$ with the property that $f p_{0}=g p_{1}$, and with the universal property that for any diagram $L \stackrel{h}{\leftarrow} X \stackrel{j}{\rightarrow} N$ that satisfies $f h=g j$, there exists a unique morphism $i: X \rightarrow L \times_{M} N$ such that $h=p_{0} i$ and $j=p_{1} i$. When a fibre product exists, it is unique up to a unique isomorphism, so in that case we may speak of the fibre product of $L$ and $N$ over $M$. In the category Grp of groups, the fibre product of $L \stackrel{f}{\rightarrow} M \stackrel{g}{\leftarrow} N$ exists, and it is given by

$$
L \times{ }_{M} N=\{(l, n) \in L \times N: f(l)=g(n)\},
$$

with $p_{0}$ and $p_{1}$ being the projection maps to $L$ and $N$, respectively.

Throughout this section $\mathcal{C}$ will denote a category in which for every diagram $L \stackrel{f}{\rightarrow} M \stackrel{g}{\leftarrow} N$ the fibre product of $L$ and $N$ over $M$ exists, equipped 
with a functor $\mathcal{C} \rightarrow$ Grp that preserves fibre products. The main examples we have in mind are the category of groups with the identity functor, the category of rings with the functor that sends a ring to its underlying additive group, and the category of finitely generated left $R$-modules for a left-noetherian ring $R$, with the functor that sends an $R$-module to its underlying abelian group.

An isogeny in $\mathcal{C}$ is a morphism that becomes an isogeny in Grp. A commensurability in $\mathcal{C}$ is a correspondence in $\mathcal{C}$ that becomes a commensurability in Grp. We will often think of an isogeny $f: L \rightarrow M$ as a special case of a commensurability, which we will denote by $c_{f}$, namely $c_{f}=(L, \mathrm{id}, f): L \rightleftharpoons M$.

The index $\mathrm{i}(f)$ of an isogeny $f$ in $\mathcal{C}$ is defined to be the index of the image of $f$ in $\mathbf{G r p}$, and the index of a commensurability is defined analogously, as in the introduction.

For each of the results $2.1-2.6$ below, it will be clear that it holds for $\mathcal{C}$ if it holds for Grp. We will therefore tacitly assume that $\mathcal{C}=\operatorname{Grp}$ in the proofs of those results.

Proposition 2.1. Let $L, M, N$ be objects in $\mathcal{C}$ and let $h$ be the composition of two morphisms $L \stackrel{f}{\rightarrow} M \stackrel{g}{\rightarrow} N$. If two of $f, g, h$ are isogenies, then so is the third. Moreover, we then have $\mathrm{i}(h)=\mathrm{i}(g) \mathrm{i}(f)$.

Proof. We have an exact sequence of pointed sets

$$
1 \rightarrow \operatorname{ker} f \rightarrow \operatorname{ker} h \rightarrow \operatorname{ker} g \rightarrow M / f L \rightarrow N / h L \rightarrow N / g M \rightarrow 1,
$$

in which each map has the property that all its non-empty fibres have equal cardinality. Hence, any term that sits between two finite sets in the above sequence is itself finite. The first assertion of the proposition easily follows. Moreover, if all terms in the sequence are finite, then the alternating product of their cardinalities is 1 , which proves the second assertion.

Definition 2.2. Let $c=(X, f, g): L \rightleftharpoons M$ and $d=(Y, h, j): M \rightleftharpoons N$ be correspondences in $\mathcal{C}$. We define the composition of $c$ with $d$ by

$$
d \circ c=\left(X \times_{M} Y, f \circ p_{0}, j \circ p_{1}\right): L \rightleftharpoons N,
$$

where $p_{0}, p_{1}$ are the canonical morphisms from $X \times_{M} Y$ to $X$, respectively $Y$.

Remark 2.3. It follows from the universal property of fibre products, and a routine diagram chase, that composition of correspondences is associative up to canonical isomorphism.

Proposition 2.4. Let $X \stackrel{g}{\rightarrow} M \stackrel{h}{\leftarrow} Y$ be morphisms in $\mathcal{C}$, and suppose that $h$ is an isogeny. Let $\left(W=X \times_{M} Y, p_{0}, p_{1}\right)$ be the fibre product of $X$ and $Y$ over $M$. Then:

(a) the morphism $p_{0}$ is an isogeny;

(b) if the image of $g$ in Grp has finite kernel, then so does the image of $p_{1}$

(c) if $g$ is an isogeny, then so is $p_{1}$. 
Proof. We first prove part (a). We have

$$
\operatorname{ker} p_{0}=\{(1, y) \in X \times Y: h(y)=g(1)=1\} \cong \operatorname{ker} h,
$$

which is finite by assumption. Further, the kernel of $g: X \rightarrow M / h Y$ is equal to $p_{0} W$, so $\left(X: p_{0} W\right) \leq(M: h Y)$, which is also finite. So $p_{0}$ is an isogeny.

Similarly, $\operatorname{ker} p_{1} \cong \operatorname{ker} g$, which proves part (b). Finally, part (c) is symmetric in $X$ and $Y$, and so follows from part (a).

Definition 2.5. A skew correspondence is a correspondence $c=(X, f, g)$ in which $f$ is an isogeny.

Proposition 2.6. If $c: L \rightleftharpoons M$ and $d: M \rightleftharpoons N$ are skew correspondences, respectively commensurabilities, then $d \circ c: L \rightleftharpoons N$ is a skew correspondence, respectively a commensurability. Moreover, if $c$ and $d$ are commensurabilities, then we have

$$
\mathrm{i}(d \circ c)=\mathrm{i}(d) \mathrm{i}(c) .
$$

Proof. The first two assertions follow immediately from Propositions 2.4 and 2.1. The third one follows from Proposition 2.1 and a routine diagram chase, which we leave to the reader.

We will now use skew correspondences in order to construct a category $\mathcal{C}_{\text {skew }}$ in which all isogenies are invertible. One can show that the class $\mathcal{I}$ of isogenies in our category $\mathcal{C}$ "admits a calculus of right fractions" in the language of Gabriel and Zisman [7, Chapter I, Section 2]; our $\mathcal{C}_{\text {skew }}$ is nothing but their "category $\mathcal{C}\left[\mathcal{I}^{-1}\right]$ of fractions".

Definition 2.7. Let $c=(X, f, g): L \rightleftharpoons M$ and $d=(Y, h, j): L \rightleftharpoons M$ be two correspondences. We say that $c$ and $d$ are equivalent if there exists a commensurability $(W, p, q): X \rightleftharpoons Y$ such that $f p=h q$ and $g p=j q$. We will call such a commensurability an equivalence between $c$ and $d$.

Proposition 2.8. Being equivalent in the sense of Definition 2.7 is an equivalence relation.

Proof. The relation is clearly symmetric. Reflexivity is also clear, since an equivalence between $(X, f, g)$ and itself is given by $(X, \mathrm{id}, \mathrm{id}): X \rightleftharpoons X$. Transitivity follows from Proposition 2.6.

Note that Definition 2.7 describes the smallest equivalence relation on the set of correspondences $L \rightleftharpoons M$ for which $(X, f, g)$ is equivalent to $(W, f p, g p)$ whenever $p: W \rightarrow X$ is an isogeny.

Definition 2.9. The inverse of a correspondence $c=(X, f, g): L \rightleftharpoons M$ is defined to be $c^{-1}=(X, g, f): M \rightleftharpoons L$.

Lemma 2.10. Let $c, c^{\prime}: L \rightleftharpoons M$ and $d: M \rightleftharpoons N$ be correspondences. Then:

(a) the correspondence $(d \circ c)^{-1}: N \rightleftharpoons L$ is equivalent to the composition $c^{-1} \circ d^{-1}$

(b) if $c$ is equivalent to $c^{\prime}$, then $c^{-1}: M \rightleftharpoons L$ is equivalent to $\left(c^{\prime}\right)^{-1}$.

Proof. The proof is easy, and is left to the reader.

Proposition 2.11. Let $c, c^{\prime}: L \rightleftharpoons M$ and $d, d^{\prime}: M \rightleftharpoons N$ be correspondences. Suppose that $c$ is equivalent to $c^{\prime}$, and $d$ is equivalent to $d^{\prime}$. Then $d \circ c$ is equivalent to $d^{\prime} \circ c^{\prime}$. 
Proof. Let $c=(X, f, g), d=(Y, h, j)$.

First, we prove the proposition in the special case that $d^{\prime}=d$, and $c^{\prime}=$ $(W, f p, g p)$, where $p: W \rightarrow X$ is an isogeny. Let $\left(X \times_{M} Y, p_{0}, p_{1}\right)$ be the fibre product of the diagram $X \stackrel{g}{\rightarrow} M \stackrel{h}{\leftarrow} Y$, and let $\left(W \times_{M} Y, p_{0}^{\prime}, p_{1}^{\prime}\right)$ be the fibre product of the diagram $W \stackrel{g p}{\rightarrow} M \stackrel{h}{\leftarrow} Y$. Thus $d \circ c=\left(X \times_{M} Y, f p_{0}, j p_{1}\right)$, and $d \circ c^{\prime}=\left(W \times_{M} Y, f p p_{0}^{\prime}, j p_{1}^{\prime}\right): L \rightleftharpoons N$. Since $g p p_{0}^{\prime}=h p_{1}^{\prime}$, the universal property of fibre products guarantees the existence of a unique map $i: W \times_{M}$ $Y \rightarrow X \times{ }_{M} Y$ with the property that $p p_{0}^{\prime}=p_{0} i$ and $p_{1}^{\prime}=p_{1} i$ :

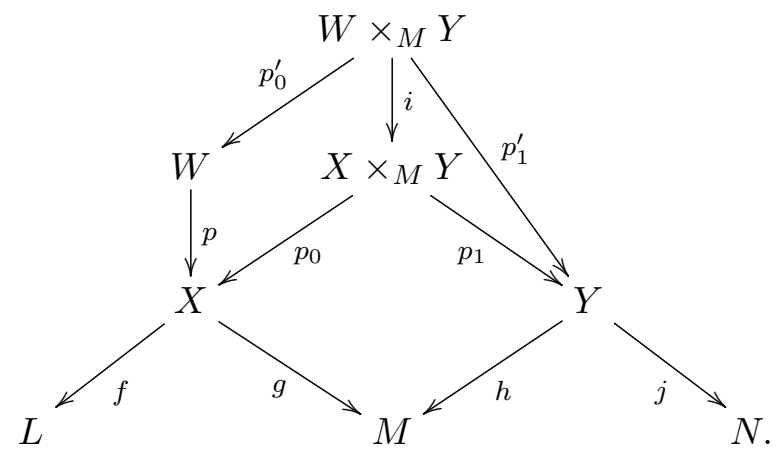

Moreover, it is easy to see that $\left(W \times_{M} Y, p_{0}^{\prime}, i\right)$ is the fibre product of the diagram $W \stackrel{p}{\rightarrow} X \stackrel{p_{0}}{\leftarrow} X \times_{M} Y$. It follows from Proposition 2.4 that $i$ is an isogeny, which proves that $d \circ c$ is equivalent to $d \circ c^{\prime}$.

Now, we prove the proposition in the special case that $d=d^{\prime}$, and $c^{\prime}$ is arbitrary. Write $\sim$ for the equivalence relation between correspondences. Let $c^{\prime}=\left(X^{\prime}, f^{\prime}, g^{\prime}\right)$, and let $(W, p, q): X \rightleftharpoons X^{\prime}$ be an equivalence between $c$ and $c^{\prime}$. Since $p$ is an isogeny, we have $c \sim(W, f p, g p)=\left(W, f^{\prime} q, g^{\prime} q\right)$, and since $q$ is an isogeny, we have $\left(W, f^{\prime} q, g^{\prime} q\right) \sim c^{\prime}$. We deduce from the special case of the proposition that we just proved that $d \circ c \sim d \circ(W, f p, g p) \sim d \circ c^{\prime}$.

Now, we prove the general case. By Lemma 2.10 and by the special case we just proved, we have

$$
(d \circ c)^{-1} \sim c^{-1} \circ d^{-1} \sim c^{-1} \circ\left(d^{\prime}\right)^{-1} \sim\left(d^{\prime} \circ c\right)^{-1} .
$$

It therefore follows from Lemma 2.10(b), that $d \circ c \sim d^{\prime} \circ c$. By the special case of the proposition that we proved already, we also have $d^{\prime} \circ c \sim d^{\prime} \circ c^{\prime}$, and the proposition follows.

Proposition 2.12. If $c$ and $d$ are two equivalent commensurabilities, then $\mathrm{i}(c)=\mathrm{i}(d)$.

Proof. This is an immediate consequence of Proposition 2.1.

The term "inverse" is justified by the following result.

Proposition 2.13. Given a commensurability $c=(X, f, g): L \rightleftharpoons M$, the composition $c^{-1} \circ c: L \rightleftharpoons L$ is equivalent to the commensurability ( $L$, id, id), and the composition $c \circ c^{-1}: M \rightleftharpoons M$ is equivalent to the commensurability $(M, \mathrm{id}, \mathrm{id})$.

Proof. First, we prove the assertion on $c^{-1} \circ c$. By definition, $c^{-1} \circ c=$ $\left(X \times_{M} X, f p_{0}, f p_{1}\right): L \rightleftharpoons L$, where $\left(X \times_{M} X, p_{0}, p_{1}\right)$ is the fibre product of the diagram $X \stackrel{g}{\rightarrow} M \stackrel{g}{\leftarrow} X$. By the universal property of the fibre product, 
we have a unique map $i: X \rightarrow X \times_{M} X$ with the property that $p_{0} i=p_{1} i=$ id: $X \rightarrow X$.

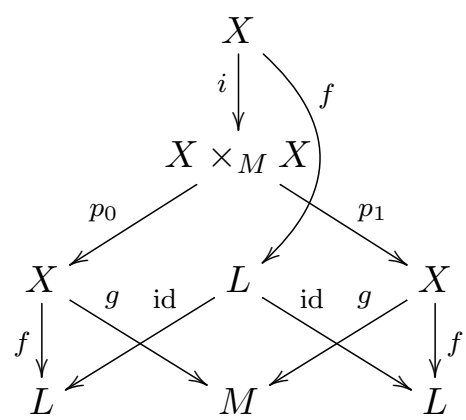

Since $g$ is an isogeny, it follows from Proposition 2.4 that $p_{0}$ is an isogeny. By Proposition 2.1, the morphism $i$ is also an isogeny, so $(X, i, f): X \times{ }_{M} X \rightleftharpoons L$ defines an equivalence between $c^{-1} \circ c$ and $(L$, id, id).

The claim for $c \circ c^{-1}$ follows by applying the result just proved to $c^{-1}$ in place of $c$.

Definition 2.14. We define $\mathcal{C}_{\text {skew }}$ to be the category with the same objects as in $\mathcal{C}$, and where, for objects $L, M$, the morphisms from $L$ to $M$ are the equivalence classes of skew correspondences $L \rightleftharpoons M$. We also define $\mathcal{C}_{\text {com }}$ to be the category with the same objects, and where the morphisms from $L$ to $M$ are the equivalence classes of commensurabilities $L \rightleftharpoons M$. It follows from Remark 2.3 and Propositions 2.8 and 2.11, that these are indeed categories, i.e. that composition of morphisms is well-defined and associative.

Proposition 2.13 implies that $\mathcal{C}_{\text {com }}$ is a (generally large) groupoid, i.e. every morphism in $\mathcal{C}_{\text {com }}$ is an isomorphism. In fact, we have the following sharper result.

Proposition 2.15. The category $\mathcal{C}_{\mathrm{com}}$ is the maximal subgroupoid of $\mathcal{C}_{\text {skew }}$.

Proof. Let $c=(X, f, g): L \rightleftharpoons M$ be a skew correspondence, and let $d=$ $(Y, h, j): M \rightleftharpoons L$ be a two-sided inverse in $\mathcal{C}_{\text {skew. }}$. So $d \circ c$ is equivalent to the commensurability ( $L$, id, id) : $L \rightleftharpoons L$, while $c \circ d$ is equivalent to ( $M$, id, id) $: M \rightleftharpoons M$, and in particular both compositions are commensurabilities. We wish to prove that $g$ is then necessarily an isogeny, and for this it is enough to assume that $\mathcal{C}=\mathbf{G r p}$.

Let $\left(Y \times_{L} X, p_{0}, p_{1}\right)$ be the fibre product of the diagram $Y \stackrel{j}{\rightarrow} L \stackrel{f}{\leftarrow} X$, and let $\left(X \times_{M} Y, p_{0}^{\prime}, p_{1}^{\prime}\right)$ be the fibre product of the diagram $X \stackrel{g}{\rightarrow} M \stackrel{h}{\leftarrow} Y$. Since $c \circ d$ is a commensurability, the morphism $g p_{1}$ is an isogeny, so $(M: g X)$ is finite. Also, since $d \circ c$ is a commensurability, the morphism $j p_{1}^{\prime}$ is an isogeny, so $\operatorname{ker} p_{1}^{\prime}$ is finite. But $\operatorname{ker} p_{1}^{\prime}=\{(x, 1) \in X \times Y: g(x)=h(1)=1\} \cong \operatorname{ker} g$. So $g$ is an isogeny, as claimed.

Theorem 2.16. Let $L$ be an object in $\mathcal{C}$. Then, the set $G_{L}$ of equivalence classes of commensurabilities $L \rightleftharpoons L$ forms a group under composition, and the map i induces a group homomorphism $G_{L} \rightarrow \mathbb{Q}_{>0}$.

Proof. The first assertion follows from the fact that $G_{L}=\operatorname{Hom}_{\mathcal{C}_{\text {com }}}(L, L)$. The second assertion follows from Propositions 2.6 and 2.12. 


\section{RING ISOGENIES}

In the present section we prove that an isogeny of rings induces an isogeny of multiplicative groups.

We begin by recalling some standard ring theoretic facts, which can be found in $[8]$.

Definition 3.1. The Jacobson radical of a ring $E$, denoted by $\mathrm{J}(E)$, is the intersection of the maximal left ideals of $R$.

Proposition 3.2. Let $E$ be a ring, and $y \in E$. Then the following are equivalent:

(a) $y \in \mathrm{J}(E)$;

(b) $y$ is contained in every maximal right ideal of $E$;

(c) y annihilates every simple left E-module;

(d) y annihilates every simple right E-module;

(e) $1-x y z \in E^{\times}$for all $x, z \in E$.

Proof. See $[8, \S 4]$.

Lemma 3.3. Let $I$ be a two-sided ideal of $E$ with $I \subset \mathrm{J}(E)$. Then the map $E^{\times} \rightarrow(E / I)^{\times}$is surjective. Moreover, $u \in E$ is a unit if and only if $u+I$ is a unit in $E / I$.

Proof. Let $u+I$ be a unit in $E / I$, and let $v+I$ be its inverse. Then we have $u v, v u \in 1+I \subset 1+\mathrm{J}(E) \subset E^{\times}$, so $u$ has both a right inverse, namely $v(u v)^{-1}$, and a left inverse, namely $(v u)^{-1} v$. It follows that $u$ is a unit in $E$.

A ring is called simple if it has exactly two two-sided ideals. A ring $E$ is called semisimple if all short exact sequences of left $E$-modules split.

Any semisimple ring is a finite direct product of simple rings. If $E$ is a semisimple ring, then the opposite ring $E^{\text {opp }}$ is also semisimple. A leftartinian ring is semisimple if and only if its Jacobson radical is 0 . If $E$ is an arbitrary ring, then $\mathrm{J}(E / \mathrm{J}(E))=0$. In particular, if $E$ is left-artinian, then $E / \mathrm{J}(E)$ is semisimple. All these facts can be found in [8, $\S 3$ and $\S 4]$.

The next lemma is also proved as [10, Lemma 2.6]. We give an alternative proof.

Lemma 3.4. Let $E$ and $F$ be rings, let $E \rightarrow F$ be a surjective ring homomorphism, and suppose that $E$ is left-artinian. Then the induced group homomorphism $E^{\times} \rightarrow F^{\times}$is surjective.

Proof. First, suppose that $E$ is semisimple. Then $E$ can be written as the product of finitely many simple rings. Since the kernel of $E \rightarrow F$ is a twosided ideal of $E$, it must be a subproduct, and $F$ may then be identified with the complementary subproduct. Surjectivity of $E^{\times} \rightarrow F^{\times}$is now obvious.

We pass to the general case. Denote by $I$ the image of $\mathrm{J}(E)$ in $F$, which is a two-sided ideal of $F$. The map $E \rightarrow F$ induces a surjective ring homomorphism $E / \mathrm{J}(E) \rightarrow F / I$, where the ring $E / \mathrm{J}(E)$ is semisimple, so by the first part of the proof the induced map $(E / \mathrm{J}(E))^{\times} \rightarrow(F / I)^{\times}$is surjective. By Lemma 3.3, the map $E^{\times} \rightarrow(E / \mathrm{J}(E))^{\times}$is also surjective, so the map $E^{\times} \rightarrow(F / I)^{\times}$induced by the composed ring homomorphism $E \rightarrow F / I$ 
is surjective as well. Now let $v \in F^{\times}$, and choose $u \in E^{\times}$that maps to $v+I \in(F / I)^{\times}$. Then the image $w$ of $u$ in $F$ satisfies $w \equiv v \bmod I$, so $w^{-1} v$ belongs to the image $1+I$ of $1+\mathrm{J}(E)$ in $F$. Let $x \in 1+\mathrm{J}(E)$ map to $w^{-1} v$. Then $u x$ maps to $w w^{-1} v=v$, and we have $u x \in E^{\times}$because $u \in E^{\times}$and $x \in 1+\mathrm{J}(E) \subset E^{\times}$. This proves surjectivity of $E^{\times} \rightarrow F^{\times}$, as required.

Lemma 3.5. Let $E$ be a ring, and let $I, J \subset E$ be two-sided ideals. Then the kernel of the natural ring homomorphism $E \rightarrow(E / I) \times(E / J)$ equals $I \cap J$, and its image is the fibre product $E / I \times_{E /(I+J)} E / J$.

The proof is straightforward, and is left to the reader.

Lemma 3.6. Let $E \rightarrow F$ be a surjective ring isogeny. Then the induced group homomorphism $E^{\times} \rightarrow F^{\times}$is surjective.

Proof. Let $I$ be the kernel of the isogeny $E \rightarrow F$. Then $I$ is finite, and we may identify $F$ with $E / I$. Write End $I$ for the endomorphism ring of the additive group of $I$. Let $J$ and $R$, respectively, be the kernel and the image of the ring homomorphism $E \rightarrow$ End $I$ sending $a \in E$ to the map $x \mapsto a x$. Then $R$, being a subring of End $I$, is a finite ring, $J$ is a two-sided ideal of $E$, and we have a ring isomorphism $E / J \rightarrow R$. By Lemma 3.5, the combined map $E \rightarrow F \times R$ induces a ring isomorphism $\varphi: E /(I \cap J) \rightarrow F \times_{E /(I+J)} R$. Now we first prove that the map $(E /(I \cap J))^{\times} \rightarrow F^{\times}$is surjective. Let $u \in F^{\times}$. Write $v$ for the image of $u$ in $(E /(I+J))^{\times}$. Since $R$ is finite and hence left-artinian, by Lemma 3.4 we can choose $w \in R^{\times}$mapping to $v \in(E /(I+J))^{\times}$. Then $(u, w)$ belongs to $F^{\times} \times_{(E /(I+J))} \times R^{\times}=\left(F \times_{E /(I+J)} R\right)^{\times}$, so $\varphi^{-1}(u, w)$ is a unit of $E /(I \cap J)$ that maps to $u \in F^{\times}$. This proves that $(E /(I \cap J))^{\times} \rightarrow F^{\times}$ is surjective. From $(I \cap J) \cdot(I \cap J) \subset J I=0$ it follows that for each $x \in I \cap J$ the element $1+x$ has inverse $1-x$ and therefore belongs to $E^{\times}$; this implies $I \cap J \subset \mathrm{J}(E)$, so by Lemma 3.3 the map $E^{\times} \rightarrow(E /(I \cap J))^{\times}$is surjective. The composed map $E^{\times} \rightarrow F^{\times}$is then surjective as well.

Part (a) of the following lemma also appears as [11, Lemma 1]. We give a new proof here.

Lemma 3.7. Let $E$ be a subring of a ring $F$ such that the index $(F: E)$ of additive groups is finite. Then:

(a) the ring $F$ has a two-sided ideal $I$ with $I \subset E$ for which the ring $F / I$ is finite;

(b) the index $\left(F^{\times}: E^{\times}\right)$is finite.

Proof. (a) Put $I=\{x \in F: F x F \subset E\}$. Then $I$ is a two-sided ideal of $F$ that is contained in $E$, and we proceed to show that $I$ has finite index in $F$. Put $J=\{x \in F: F x \subset E$ and $x F \subset E\}$. Then we have $I \subset J \subset E \subset F$. Denote by $D$ the finite abelian group $F / E$, by End $D$ its endomorphism ring, and by (End $D)^{\text {opp }}$ the ring opposite to End $D$. Both of these rings are finite. The natural left and right $E$-module structures on $D$ induce a ring homomorphism $E \rightarrow($ End $D) \times(\text { End } D)^{\text {opp }}$ of which $J$ is the kernel. It follows that $J$ is a two-sided ideal of $E$ of finite index in $E$. There is a well-defined group homomorphism

$$
\begin{aligned}
J & \rightarrow \operatorname{Hom}\left(D \otimes_{\mathbb{Z}} D, D\right) \\
x & \mapsto((y+E) \otimes(z+E) \mapsto y x z+E)
\end{aligned}
$$


for $x \in J, y, z \in F$. Its kernel is $I$, and since $D \otimes_{\mathbb{Z}} D$ is finite, the group $J / I$ is finite. Because each of $F / E, E / J, J / I$ is finite, the ring $F / I$ is finite. This proves (a).

(b) Let $I$ be as in (a). Then $F / I$ and $(F / I)^{\times}$are finite, so the kernel $K$ (say) of the natural group homomorphism $F^{\times} \rightarrow(F / I)^{\times}$has finite index in $F^{\times}$. If $x \in K$, then $x^{-1} \in K$, so both $x$ and $x^{-1}$ are in $1+I$, which is contained in $E$. This proves $K \subset E^{\times}$, so $E^{\times}$has finite index in $F^{\times}$as well. This proves (b).

We can now prove Theorem 1.3 of the introduction, which reads as follows.

Theorem 3.8. Let $E \rightarrow F$ be a ring isogeny. Then the induced group homomorphism $E^{\times} \rightarrow F^{\times}$is a group isogeny. If in addition the map $E \rightarrow F$ is surjective, then so is the induced map $E^{\times} \rightarrow F^{\times}$.

Proof. The last assertion is Lemma 3.6. For the first assertion, let $I$ and $D$ be the kernel, respectively the image of the map $E \rightarrow F$. Then the kernel of $E^{\times} \rightarrow D^{\times}$is contained in $1+I$ and therefore finite, and by Lemma 3.6 the image is all of $D^{\times}$. Hence $E^{\times} \rightarrow D^{\times}$is a group isogeny. Further, the inclusion map $D^{\times} \rightarrow F^{\times}$is obviously injective, while by Lemma 3.7(b) the index of $D^{\times}$in $F^{\times}$is finite. Hence $D^{\times} \rightarrow F^{\times}$is a group isogeny. By Proposition 2.1, the composed map $E^{\times} \rightarrow F^{\times}$is also a group isogeny.

\section{Residually Finite Domains}

This section is devoted to some properties of infinite domains all of whose proper quotients are finite.

Lemma 4.1. Let $Z$ be a domain such that for all non-zero $m \in Z$ the ring $Z / m Z$ is finite, let $Q$ be the field of fractions of $Z$, let $V$ be a finite dimensional $Q$-vector space, and let $L$ be a sub-Z-module of $V$. Then for all nonzero $m \in Z$ the $Z$-module $L / m L$ is finite of order dividing $\#(Z / m Z)^{\operatorname{dim}_{Q} V \text {, }}$ with equality if $L$ is finitely generated and $Q \cdot L=V$.

Proof. Let $m \in Z$ be non-zero. First suppose that $L$ is finitely generated. Let $S \subset L$ be a finite subset that generates it as a $Z$-module, let $T \subset S$ be a maximal subset that is linearly independent over $Q$, and let $M \subset L$ be the $Z$-module generated by $T$. Then $M$ is $Z$-free of rank \#T, so $M / m M$

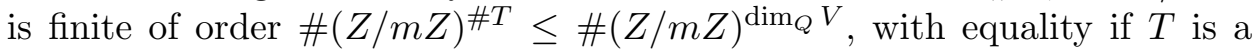
$Q$-basis of $V$ or, equivalently, if $Q \cdot L=V$. By maximality of $T$, we can, for each $s \in S$, choose a non-zero element $m_{s} \in Z$ such that $m_{s} s \in M$, and $m^{\prime}=\prod_{s \in S} m_{s}$ is then a non-zero element of $Z$ satisfying $m^{\prime} L \subset M$. Because $M / m^{\prime} M$ is finite, its subgroup $m^{\prime} L / m^{\prime} M$ is finite as well, and since the latter group is isomorphic to $L / M$ and to $m L / m M$, we find that $L / M$ and $m L / m M$ are finite of the same order. The group $L / m M$ is finite of order \# $(L / M) \cdot \#(M / m M)$, so $L / m L$ is also finite, of order

$$
\frac{\#(L / M) \cdot \#(M / m M)}{\#(m L / m M)}=\#(M / m M)=\#(Z / m Z)^{\# T} \leq \#(Z / m Z)^{\operatorname{dim}_{Q} V},
$$

with equality if $Q \cdot L=V$.

Passing to the general case, let $U$ be the set of finitely generated sub- $Z$ modules $L^{\prime}$ of $L$, which is a directed partially ordered set by inclusion. Then 
$L$ is the injective limit of all $L^{\prime} \in U$, and $L / m L$ is the injective limit of the modules $L^{\prime} / m L^{\prime}$, all of which have order dividing $\#(Z / m Z)^{\operatorname{dim}_{Q} V}$. The injective limit has then also order dividing the same number. This completes the proof of Lemma 4.1.

We now prove Theorem 1.4.

Theorem 4.2. Let $Z$ be a domain such that for all non-zero $m \in Z$ the ring $Z / m Z$ is finite, let $Q$ be the field of fractions of $Z$, let $A$ be a semisimple $Q$-algebra of finite vector space dimension over $Q$, and let $R \subset A$ be a sub$Z$-algebra with $Q \cdot R=A$. Then $R$ is left-noetherian and right-noetherian.

Proof. Let $I$ be a left ideal of $R$. Then $Q \cdot I$ is a left $A$-ideal, so by semisimplicity of $A$ it is a direct summand of the left $A$-module $A$. Thus the endomorphism ring of the latter module contains an idempotent with image $Q \cdot I$. Since the endomorphisms of the left $A$-module $A$ are the right multiplications by elements of $A$, it is equivalent to say that we can choose an idempotent $e \in A$ with $A e=Q \cdot I$. We have $e \in Q \cdot I$, so we can choose a non-zero element $m \in Z$ with $m e \in I$. Multiplying the chain of inclusions $R m e \subset I \subset R$ by $e$ on the right, which when restricted to $I$ is just the identity map, we obtain $R m e \subset I \subset R e$, where $R m e=m R e$ because $m$ is central. By Lemma 4.1, the group Re/mRe is finite, so $I / R m e$ is finite as well. Hence $I$ is, as a left $R$-module, generated by me together with a finite set, and is therefore finitely generated. This proves that $R$ is left-noetherian. Applying this result to $A^{\text {opp }}$ and $R^{\text {opp }}$, we find that $R$ is right-noetherian as well.

Example 4.3. If we assume $Z \neq Q$, then the semisimplicity condition on $A$ is actually necessary for the conclusion of Theorem 4.2 to be valid for all $R$. To see this, assume that $A$ is not semisimple, or equivalently that $\mathrm{J}(A) \neq 0$, and choose a sub- $Z$-algebra $T \subset A$ that is finitely generated as a $Z$-module and satisfies $Q \cdot T=A$. Then the ring $R=T+\mathrm{J}(A)$ is not leftnoetherian because $\mathrm{J}(A)$ is not finitely generated as a left $R$-ideal. If it were, then the non-zero $Q$-vector space $\mathrm{J}(A) / \mathrm{J}(A)^{2}$ would be finitely generated as a $T$-module and hence as a $Z$-module, which for $Z \neq Q$ is impossible.

Lemma 4.4. Let $Z$ be an infinite commutative ring. Suppose that there exists a faithful $Z$-module $M$ with the property that for all non-zero $m \in Z$, $M / m M$ is finite. Then $Z$ is a domain.

Proof. Let $a, b \in Z$ be non-zero. We have an exact sequence of $Z$-modules

$$
M / b M \stackrel{a}{\rightarrow} M / a b M \rightarrow M / a M \rightarrow 0 .
$$

The left and right terms are finite by assumption, so $M / a b M$ is finite. But since $Z$ is infinite, and $M$ is a faithful module, $M$ is also infinite, and so $a b \neq 0$.

The following result gives a description of the rings $Z$ that occur in Theorem 1.2 .

Theorem 4.5. Let $Z$ be an infinite commutative ring. Then the following assertions are equivalent:

(a) for each non-zero $m \in Z$, the ring $Z / m Z$ is finite; 
(b) the ring $Z$ is a domain, and each non-zero prime ideal $\mathfrak{p}$ of $Z$ is finitely generated as an ideal and has finite index in $Z$;

(c) either $Z$ is a field, or it is a one-dimensional noetherian domain with the property that for every maximal ideal $\mathfrak{m}$ of $Z$ the field $Z / \mathfrak{m}$ is finite.

Proof. First we prove that (a) implies (b). From (a) it follows, by Lemma 4.4 applied to $M=Z$, that $Z$ is a domain. Now let $\mathfrak{p}$ be a non-zero prime ideal, and let $m \in \mathfrak{p}$ be non-zero. Then we have $m Z \subset \mathfrak{p} \subset Z$, and since $Z / m Z$ is finite, the index of $\mathfrak{p}$ in $Z$ is finite and $\mathfrak{p} / m Z$ is finite. Hence $\mathfrak{p}$ is generated by $m$ together with a finite set, and is therefore finitely generated.

Now we prove that (b) implies (c). By [5, Theorem 2], each commutative ring of which every prime ideal is finitely generated is noetherian. Hence (b) implies that $Z$ is noetherian. If $\mathfrak{p}$ is a non-zero prime ideal, then $Z / \mathfrak{p}$ is a finite domain, and therefore a field. Hence each non-zero prime ideal is maximal, so $Z$ has Krull dimension 0 or 1 ; in the former case it must be a field.

Finally, suppose that (c) holds. Then, we will deduce (a) by showing that for any non-zero ideal $I$ of $Z$, the ring $Z / I$ is finite. Suppose that there exists a non-zero ideal $I$ in $Z$ such that $Z / I$ is infinite. Since $Z$ is noetherian, we may, without loss of generality, assume that $I$ is maximal among ideals with this property. So $Z / I$ is infinite, but its quotient by any non-zero ideal is finite. It follows from Lemma 4.4, applied to $M=Z / I$, that $Z / I$ is a domain, so $I$ is a prime ideal of $Z$. It is also non-zero, so (c) implies that $I$ is maximal, and therefore that $Z / I$ is finite, which is a contradiction.

\section{ON THE UNITS OF SEMISIMPLE RINGS}

By a division ring we mean a ring $D$ with the property $D^{\times}=D \backslash\{0\}$. If $D$ is a division ring and $n$ is a positive integer, then $\mathrm{M}(n, D)$ denotes the ring of $n$ by $n$ matrices over $D$. If $G$ is a group, then $G_{\mathrm{ab}}$ denotes the maximal abelian quotient of $G$.

Lemma 5.1. Let $n$ be a positive integer, let $D$ be a division ring, and for $x \in D^{\times}$and $j \in\{1, \ldots, n\}$, let $\delta_{j}(x) \in \mathrm{M}(n, D)$ be the diagonal matrix with $j$ th entry equal to $x$ and all other entries equal to 1 . Then each map $\delta_{j}$ is a group homomorphism $D^{\times} \rightarrow \mathrm{M}(n, D)^{\times}$, they all induce the same group homomorphism $\bar{\delta}: D_{\mathrm{ab}}^{\times} \rightarrow \mathrm{M}(n, D)_{\mathrm{ab}}^{\times}$, and if $n \neq 2$ or $\# D \neq 2$ then $\bar{\delta}$ is surjective.

Proof. It is clear that each $\delta_{j}$ is a group homomorphism, and that for each $x$ all $\delta_{j}(x)$ are conjugate to each other, so all $\delta_{j}$ induce the same map $D_{\mathrm{ab}}^{\times} \rightarrow$ $\mathrm{M}(n, D)_{\mathrm{ab}}^{\times}$. It is evidently surjective if $n=1$.

For $i, j \in\{1, \ldots, n\}, i \neq j$, and $x \in D$, let $B_{i j}(x) \in \mathrm{M}(n, D)$ be the matrix obtained from the unit matrix by replacing the $(i, j)$-entry by $x$; then one has $B_{i j}(x) \in \mathrm{M}(n, D)^{\times}$. The subgroup of $\mathrm{M}(n, D)^{\times}$generated by all $B_{i j}(x)$ is denoted by $\operatorname{SL}_{n}(D)$.

By $\left[1\right.$, Chapter IV, Theorem 4.1], we have $\mathrm{M}(n, D)^{\times}=\mathrm{SL}_{n}(D) \cdot \delta_{n}\left(D^{\times}\right)$. Each $B_{i j}(x)$ is a transvection of the right $D$-vector space $D^{n}$ in the sense of [1, Chapter IV, Definition 4.1]. Assume now that $n>2$ or $\# D \neq 2$. Then by $\left[1\right.$, Chapter IV, Section 2] each transvection belongs to $\left[\mathrm{M}(n, D)^{\times}, \mathrm{M}(n, D)^{\times}\right]$, 
so $\mathrm{SL}_{n}(D) \subset\left[\mathrm{M}(n, D)^{\times}, \mathrm{M}(n, D)^{\times}\right]$, and therefore

$$
\mathrm{M}(n, D)^{\times}=\left[\mathrm{M}(n, D)^{\times}, \mathrm{M}(n, D)^{\times}\right] \cdot \delta_{n}\left(D^{\times}\right) .
$$

This implies that $\bar{\delta}$ is surjective.

Lemma 5.2. Let $n$ be a positive integer, let $D$ be a division ring, and for each $x \in D^{\times}$let $\iota(x) \in \mathrm{M}(n, D)^{\times}$be $x$ times the identity matrix. Then $\iota$ is a group homomorphism $D^{\times} \rightarrow \mathrm{M}(n, D)^{\times}$, and the group

$$
\mathrm{M}(n, D)^{\times} /\left(\iota\left(D^{\times}\right) \cdot\left[\mathrm{M}(n, D)^{\times}, \mathrm{M}(n, D)^{\times}\right]\right)
$$

is abelian of exponent dividing $n$.

Proof. It is clear that $\iota$ is a group homomorphism. If we have $n=\# D=2$, then $\mathrm{M}(n, D)^{\times}$is a non-abelian group of order 6 , in which case $\mathrm{M}(n, D)_{\mathrm{ab}}^{\times}$ has order 2 and the conclusion of the lemma is valid. Assume now that $n \neq 2$ or $\# D \neq 2$, so that the map $\bar{\delta}$ from Lemma 5.1 is surjective.

Denote by $\bar{\iota}: D_{\mathrm{ab}}^{\times} \rightarrow \mathrm{M}(n, D)_{\mathrm{ab}}^{\times}$the map induced by $\iota$. For each $x \in D^{\times}$ one has $\iota(x)=\prod_{j=1}^{n} \delta_{j}(x)$ and therefore $\bar{\iota}(x)=\bar{\delta}(x)^{n}$, so the surjectivity of $\bar{\delta}$ yields

and the lemma is proved.

$$
\bar{\iota}\left(D_{\mathrm{ab}}^{\times}\right)=\bar{\delta}\left(D_{\mathrm{ab}}^{\times}\right)^{n}=\left(\mathrm{M}(n, D)_{\mathrm{ab}}^{\times}\right)^{n},
$$

The following result is due to Wedderburn [13].

Theorem 5.3. Let $D$ be a division ring with centre $\mathrm{Z}(D)$, let $a \in D$, and let $f \in \mathrm{Z}(D)[X]$ be an irreducible polynomial with leading coefficient 1 such that $f(a)=0$. Put $l=\operatorname{deg} f$. Then there exist $b_{1}, b_{2}, \ldots, b_{l} \in D^{\times}$such that in $D[X]$ one has

$$
f=\left(X-b_{1} a b_{1}^{-1}\right) \cdot \ldots \cdot\left(X-b_{l} a b_{l}^{-1}\right) .
$$

Proof. See [8, Theorem (16.9)].

Lemma 5.4. Let $D$ be a division ring that has finite vector space dimension $m^{2}$ over its centre $\mathrm{Z}(D)$, where $m$ is a positive integer. Then the group $D^{\times} /\left(\mathrm{Z}(D)^{\times} \cdot\left[D^{\times}, D^{\times}\right]\right)$is abelian of exponent dividing $m$.

Proof. Since $D^{\times} /\left(\mathrm{Z}(D)^{\times} \cdot\left[D^{\times}, D^{\times}\right]\right)$is a quotient of $D_{\mathrm{ab}}^{\times}$, it is an abelian group. Let $a \in D^{\times}$. It will suffice to show that the image $\bar{a}$ of $a$ in the quotient $D^{\times} /\left(\mathrm{Z}(D)^{\times} \cdot\left[D^{\times}, D^{\times}\right]\right)$has order dividing $m$. The subfield $\mathrm{Z}(D)(a)$ of $D$ is contained in a maximal subfield of $D$, and each maximal subfield of $D$ is an extension field of $\mathrm{Z}(D)$ of degree $m$, by $[6,(7.22)]$. Hence we have $[\mathrm{Z}(D)(a)$ : $\mathrm{Z}(D)]=l$ for some divisor $l$ of $m$, and $a$ is a zero of an irreducible polynomial $f \in \mathrm{Z}(D)[X]$ of degree $l$ with leading coefficient 1 . Using Theorem 5.3 we find $b_{1}, \ldots, b_{l} \in D^{\times}$such that $b_{1} a b_{1}^{-1} \cdot \ldots \cdot b_{l} a b_{l}^{-1}=(-1)^{l} f(0) \in \mathrm{Z}(D)^{\times}$. Mapping this identity to the abelian group $D^{\times} /\left(\mathrm{Z}(D)^{\times} \cdot\left[D^{\times}, D^{\times}\right]\right)$we obtain $\bar{a}^{l}=1$, so $\bar{a}^{m}=1$, as required. This proves Lemma 5.4.

We can now prove Theorem 1.6 and deduce Theorem 1.5. We recall the statements.

Theorem 5.5. Let $k$ be a field, and let $B$ be a central simple algebra over $k$. Let the dimension of $B$ as a vector space over $k$ be $d^{2}$, where $d$ is a positive integer. Then the group $B^{\times} /\left(k^{\times}\left[B^{\times}, B^{\times}\right]\right)$is abelian of exponent dividing $d$. 
Proof. By $[2, \S 14$, Theorem 1], there are a positive integer $n$ and a division ring $D$ with $\mathrm{Z}(D)=k$ such that $B$ is, as an algebra over $k$, isomorphic to $\mathrm{M}(n, D)$. Then $D$ has finite degree $m^{2}$ over $k$, and $n m=d$. By Lemma 5.4, the cokernel of the natural group homomorphism $k^{\times} \rightarrow D_{\mathrm{ab}}^{\times}$has exponent dividing $m$, and by Lemma 5.2 the cokernel of the natural group homomorphism $D_{\mathrm{ab}}^{\times} \rightarrow \mathrm{M}(n, D)^{\times}$has exponent dividing $n$. It follows that the cokernel of the natural group homomorphism $k^{\times} \rightarrow \mathrm{M}(n, D)_{\mathrm{ab}}^{\times}$has exponent dividing $n m=d$.

Theorem 5.6. Let $B$ be a semisimple ring that is finitely generated as a module over its centre $\mathrm{Z}(B)$. Then $B^{\times} /\left(\mathrm{Z}(B)^{\times}\left[B^{\times}, B^{\times}\right]\right)$is an abelian group of finite exponent.

Proof. In the case the semisimple ring $B$ is simple, our hypothesis that it be finite over its centre implies that it is a central simple algebra over $\mathrm{Z}(B)$, and the assertion follows from Theorem 5.5. Generally, by [8, Chapter 1, Theorem (3.5)] the ring $B$ is a product of finitely many semisimple rings that are simple, and the result follows from the case we just did.

\section{SKew CORRESPONDENCES AS MORPHISMS}

As announced in the introduction, in this section we elaborate upon an argument of Serre (see e.g. [12, Tag 0B0J]) to prove an equivalence between two categories of modules. The main result of the section is Theorem 6.5. We will need the notion of a skew correspondence (Definition 2.5), and the constructions of the categories $\mathcal{C}_{\text {skew }}$ and $\mathcal{C}_{\text {com }}$ (Definition 2.14).

Notation 6.1. The following assumptions will be in force throughout the present section: $Z$ is an infinite commutative ring that satisfies the equivalent conditions of Theorem 4.5, with field of fractions $Q$; further, $A$ is a $Q$-algebra of finite vector space dimension over $Q$, and $R$ is a left-noetherian sub- $Z$ algebra of $A$ with the property that $Q \cdot R=A$. By an $R$-module we shall always mean a left $R$-module. We call a module finite if its cardinality is finite. If $L$ is a finitely generated $R$-module, let $L_{\text {tors }}$ denote the set of all elements of $L$ that have a non-zero annihilator in $Z$. Since the image of $Z$ in $R$ is central, $L_{\text {tors }}$ is an sub- $R$-module.

We remark that the hypotheses of Section 2 on the category $\mathcal{C}$ are satisfied for the category of finitely generated $R$-modules. We will tacitly use this fact throughout the rest of the paper.

Lemma 6.2. Let $L$ be a finitely generated $R$-module, and let $U$ be a sub-Rmodule. Then $U$ is finite if and only if it is contained in $L_{\text {tors }}$.

Proof. First, we show that $L_{\text {tors }}$ is finite. Since $R$ is left-noetherian, $L_{\text {tors }}$ is finitely generated as an $R$-module. So there exists a non-zero $m \in Z$ that annihilates $L_{\text {tors }}$, and $L_{\text {tors }}$ is then a finitely generated module over the ring $R / m R$, which is finite by Lemma 4.1. This proves one implication.

For the converse, let $U \subset L$ be a finite sub-R-module. Then for each $x \in U$, the set $\{z x: z \in Z\}$ is finite, so the annihilator of $x$ in $Z$ has finite index in $Z$; in particular it is non-zero, since $Z$ is assumed to be infinite, so $x \in L_{\text {tors }}$. 
Theorem 6.3. Let $L, M$ be two finitely generated $R$-modules. Then there exists an isogeny of $R$-modules $L \rightarrow M$ if and only if there exists a commensurability of $R$-modules $L \rightleftharpoons M$, and if and only if there exists an isomorphism of $A$-modules $Q \otimes_{Z} L \cong Q \otimes_{Z} M$.

Proof. First, suppose that $f: L \rightarrow M$ is an isogeny. Then $c_{f}=(L, \mathrm{id}, f): L \rightleftharpoons$ $M$ is a commensurability.

Next, suppose that we have a commensurability $(X, f, g): L \rightleftharpoons M$. Then the kernels and cokernels of $f, g$ are finite $R$-modules, and so are $Z$-torsion modules by Lemma 6.2. They are therefore annihilated by the functor $Q \otimes_{Z}$ -, so the maps $Q \otimes_{Z} f$ and $Q \otimes_{Z} g$ are isomorphisms.

Finally, suppose that we have an isomorphism $\phi: Q \otimes_{Z} L \rightarrow Q \otimes_{Z} M$ of $A$ modules. It follows from Lemma 6.2 that the quotient map $L \rightarrow \bar{L}=L / L_{\text {tors }}$ is an isogeny. Since $\bar{L}$ is $Z$-torsion free, it embeds into $Q \otimes_{Z} L$, and similarly for $\bar{M}$. By "clearing denominators", we can find non-zero elements $m_{1}, m_{2} \in$ $Z$ such that $m_{1} \phi(\bar{L})$ is contained in $\bar{M} \subset Q \otimes_{Z} M$, and $\phi(\bar{L})$ contains $m_{2} \bar{M}$. Since $\bar{M} / m_{1} m_{2} \bar{M}$ is finite by Lemma 4.1 , it follows that $m_{1} \phi: \bar{L} \rightarrow \bar{M}$ is an isogeny. Let $m_{3} \in Z$ be a non-zero element that annihilates $M_{\text {tors }}$. Then $m_{3} M$ is canonically isomorphic to $\bar{M}$, and since $M / m_{3} M$ is finitely generated and torsion, Lemma 6.2 implies that the embedding $\bar{M} \cong m_{3} M \subset$ $M$ is an isogeny. The composition of the three isogenies $L \rightarrow \bar{L} \rightarrow \bar{M} \rightarrow M$ is an isogeny by Proposition 2.1, as claimed.

Lemma 6.4. Let $L, M$ be finitely generated $R$-modules, and let $(X, f, g)$ and $(Y, h, j): L \rightleftharpoons M$ be equivalent skew correspondences. Let $Q \otimes_{Z} f$ denote the map of $A$-modules $Q \otimes_{Z} L \rightarrow Q \otimes_{Z} M$ induced by $f$, and similarly for $g, h, j$. Then $(Q \otimes g) \circ(Q \otimes f)^{-1}=(Q \otimes j) \circ(Q \otimes h)^{-1}$.

Proof. Let $(W, p, q): X \rightleftharpoons Y$ be an equivalence between $(X, f, g)$ and $(Y, h, j)$. Since $p$ and $q$ are isogenies, Lemma 6.2 implies that $Q \otimes_{Z} p$ and $Q \otimes_{Z} q$ are both invertible. Moreover, we have

$$
\begin{aligned}
& Q \otimes_{Z} f=\left(Q \otimes_{Z} h\right) \circ\left(Q \otimes_{Z} q\right) \circ\left(Q \otimes_{Z} p\right)^{-1}, \\
& Q \otimes_{Z} g=\left(Q \otimes_{Z} j\right) \circ\left(Q \otimes_{Z} q\right) \circ\left(Q \otimes_{Z} p\right)^{-1},
\end{aligned}
$$

so $(Q \otimes g) \circ(Q \otimes f)^{-1}=(Q \otimes j) \circ(Q \otimes h)^{-1}$.

Let ${ }_{R}$ Mod, respectively ${ }_{A}$ Mod denote the category of finitely generated $R$ modules, respectively finitely generated $A$-modules. By Lemma 6.4, we may define a functor $\mathcal{F}$ from ${ }_{R} \mathbf{M o d}_{\text {skew }}$ to ${ }_{A}$ Mod by sending an $R$-module $L$ to the $A$-module $Q \otimes_{Z} L$, and an equivalence class of skew correspondences represented by $(X, f, g): L \rightleftharpoons M$ to the map of $A$-modules $(Q \otimes g) \circ(Q \otimes$ $f)^{-1}: Q \otimes_{Z} L \rightarrow Q \otimes_{Z} M$. The verification that $\mathcal{F}$ respects composition of morphisms, and thus does define a functor, is easy and is left to the reader.

Theorem 6.5. The functor $\mathcal{F}:{ }_{R} \mathbf{M o d}_{\text {skew }} \rightarrow{ }_{A} \operatorname{Mod}$ is an equivalence of categories.

To prove the theorem, we will show in the next three lemmas that the functor $\mathcal{F}$ has dense image, is full, and is faithful.

Lemma 6.6. Any element of ${ }_{A} \mathrm{Mod}$ is isomorphic to $\mathcal{F}(L)$ for some $R$ module $L$. 
Proof. Let $V$ be an $A$-module with finite generating set $S$. Let $L$ be the sub- $R$-module of $V$ generated by $S$ over $R$. Then the $A$-module $\mathcal{F}(L)$ is isomorphic to $V$.

Lemma 6.7. Let $L, M$ be finitely generated $R$-modules, and let $\phi: \mathcal{F}(L) \rightarrow$ $\mathcal{F}(M)$ be a morphism of $A$-modules. Then there exists a skew correspondence $c: L \rightleftharpoons M$ such that $\mathcal{F}(c)=\phi$.

Proof. Let $\bar{L}$ be the image of $L$ in $Q \otimes_{Z} L$, and let $\bar{M}$ be the image of $M$ in $Q \otimes_{Z} M$. By Lemma 6.2, the natural map $f: L \rightarrow Q \otimes_{Z} L$ gives rise to a commensurability $c_{L}=(L$, id, $f): L \rightleftharpoons \bar{L}$, and similarly we have a commensurability $c_{M}: M \rightleftharpoons \bar{M}$. Since $\bar{L}$ and $\bar{M}$ are finitely generated as $R$-modules, and since $\bar{M}$ generates $Q \otimes_{Z} M$ over $Q$, we may choose a non-zero $m \in Z$ such that $m \phi(\bar{L})$ is contained in $\bar{M}$. Let $g$ be the inclusion $m \phi(\bar{L}) \subset \bar{M}$, and define the correspondence $c_{\phi}=(\bar{L}, m, g m \phi): \bar{L} \rightleftharpoons \bar{M}$. It follows from Lemma 4.1 that $c_{\phi}$ is a skew correspondence. By Proposition 2.6, the composition $c=c_{M}^{-1} \circ c_{\phi} \circ c_{L}: L \rightleftharpoons M$ is also a skew correspondence, and it is easy to see that $\mathcal{F}(c)=\phi$.

Lemma 6.8. Let $L, M$ be finitely generated $R$-modules, and let $c, d: L \rightleftharpoons M$ be two skew correspondences such that $\mathcal{F}(c)=\mathcal{F}(d)$. Then $c$ and $d$ are equivalent.

Proof. Let $c=(X, f, g)$, and $d=(Y, h, j)$. We will show that $c$ and $d$ are equivalent by showing that the fibre product $\left(X \times_{L \oplus M} Y, p_{0}, p_{1}\right): X \rightleftharpoons Y$ is a commensurability.

First, assume that the images of $f, g, h$, and $j$ are $Z$-torsion free. Then $f$ and $g$ factor through $X / X_{\text {tors }}$, and similarly for $h$ and $j$. By Lemma 6.2, the quotient maps $X \rightarrow X / X_{\text {tors }}$ and $Y \rightarrow Y / Y_{\text {tors }}$ are isogenies, so after replacing $c$ and $d$ by equivalent commensurabilities, we may assume that $X$ and $Y$ are $Z$-torsion free. It then follows from Lemma 6.2 that $f, g, h$, and $j$ are injective. Since $\mathcal{F}(c)=\mathcal{F}(d)$, we have

$$
\left(Q \otimes_{Z} g\right) \circ\left(Q \otimes_{Z} f\right)^{-1}=\left(Q \otimes_{Z} j\right) \circ\left(Q \otimes_{Z} h\right)^{-1},
$$

and it follows that the canonical injection $X \times_{L \oplus M} Y \rightarrow X \times_{L} Y$ is an isomorphism. By Proposition 2.6, the fibre product $\left(X \times_{L} Y, p_{0}, p_{1}\right): X \rightleftharpoons Y$ of the diagram $X \rightarrow M \leftarrow Y$ is a commensurability, which proves this special case of the lemma.

We now prove the general case. By applying Lemma 6.2 with $U=f(X)_{\text {tors }}$, and similarly for $g, h$, and $j$, we may choose a non-zero $m \in Z$ such that the images of $m f, m g, m h$, and $m j$ are $Z$-torsion free. It is easy to see that $c$ is equivalent to $(X, m f, m g)$, and $d$ is equivalent to $(Y, m h, m j)$. So the general case follows from the special case above.

Proof of Theorem 6.5. The result follows by combining Lemmas 6.6, 6.7, and 6.8 .

Recall from Theorem 2.16 that if $L$ is a finitely generated $R$-module, we let $G_{L}$ denote the group of equivalence classes of commensurabilities $L \rightleftharpoons L$ under composition. It may be viewed as the full subgroupoid of ${ }_{R} \mathbf{M} \mathbf{M o d}_{\text {com }}$ whose only object is $L$. 
Corollary 6.9. Let $L$ be a finitely generated $R$-module. Then the map $G_{L} \rightarrow$ $\operatorname{Aut}_{A}\left(Q \otimes_{Z} L\right),(X, f, g) \mapsto(Q \otimes g) \circ(Q \otimes f)^{-1}$ is a group isomorphism.

Proof. By Proposition 2.15, the category ${ }_{R} \mathbf{M o d}{ }_{\text {com }}$ is the maximal subgroupoid of ${ }_{R} \mathbf{M o d}_{\text {skew }}$. So Theorem 6.5 implies that the functor $\mathcal{F}$ induces an equivalence of categories from ${ }_{R} \mathbf{M o d}_{\text {com }}$ to the category whose objects are the finitely generated $A$-modules, and whose morphisms are the $A$-module isomorphisms. The corollary follows by restricting $\mathcal{F}$ to the full subgroupoid $G_{L}$ of ${ }_{R} \mathbf{M o d}_{\text {skew }}$.

\section{Automorphisms of Commensurabilities}

It is in the present section that we construct ring and group commensurabilities out of module commensurabilities. Here we retain the assumptions of Notation 6.1 .

Let $c=(N, f, g): L \rightleftharpoons M$ be a correspondence of $R$-modules. In the introduction we defined the endomorphism ring of $c$ to be End $c=\{(\lambda, \nu, \mu) \in$ $($ End $L) \times($ End $N) \times($ End $M): \lambda f=f \nu, \mu g=g \nu\}$. We also recall the correspondence $\mathrm{e}(c)=\left(\right.$ End $\left.c, p_{0}, p_{1}\right):$ End $L \rightleftharpoons$ End $M$, given by sending $(\lambda, \nu, \mu) \in$ End $c$ to $\lambda$ and $\mu$, respectively, and the induced correspondence of automorphism groups a $(c)$ : Aut $L \rightleftharpoons$ Aut $M$. If $f: L \rightarrow M$ is an isogeny, we let $c_{f}$ be the commensurability $(L$, id, $f): L \rightleftharpoons M$, as in Section 2 .

Lemma 7.1. Let $f: L \rightarrow M$ be an isogeny of finitely generated $R$-modules. Then the correspondence $\mathrm{e}\left(c_{f}\right)$ : End $L \rightleftharpoons$ End $M$ is a commensurability of rings.

Proof. We first show that $p_{1}$ has finite kernel. We have

$$
\operatorname{ker} p_{1}=\{(\lambda, \lambda, 0) \in \text { End } L \times \text { End } L \times \text { End } M: f \lambda=0\} \cong \operatorname{Hom}(L, \operatorname{ker} f) \text {, }
$$

which is finite since $L$ is finitely generated and ker $f$ is finite by assumption.

Next, we show that the image of $p_{1}$ has finite additive index in End $M$. The modules $L_{\text {tors }}$ and $M / f(L)$ are finite, so by Lemma 6.2 there exist non-zero $m_{1}, m_{2} \in Z$ such that $m_{1}$ annihilates $L_{\text {tors }}$, and $m_{2}$ annihilates $M / f(L)$. Thus, $f: m_{1} L \rightarrow m_{1} M$ is injective, and the image contains $m_{1} m_{2} M$, so $f^{-1}$ defines a homomorphism $m_{1} m_{2} M \rightarrow m_{1} L$. Given $\mu \in$ End $M$, we may therefore define $\lambda: L \rightarrow L, x \mapsto f^{-1}\left(m_{1} m_{2} \mu(f(x))\right)$, which has the property that $\left(\lambda, \lambda, m_{1} m_{2} \mu\right) \in$ End $c_{f}$. So the image of $p_{1}$ contains $m_{1} m_{2}$ End $M$, which has finite additive index in End $M$ by Lemma 4.1. This proves that $p_{1}$ is an isogeny.

We now show that the image of $p_{0}$ has finite additive index in End $L$. Given any $\lambda \in$ End $L$, we may define $\mu: M \rightarrow M, y \mapsto f\left(\lambda\left(f^{-1}\left(m_{1} m_{2} y\right)\right)\right)$, where $m_{1}, m_{2}$ are as before. We then have $\left(m_{1} m_{2} \lambda, m_{1} m_{2} \lambda, \mu\right) \in$ End $c_{f}$. So the image of $p_{0}$ contains $m_{1} m_{2}$ End $L$, which has finite additive index in End $L$ by Lemma 4.1 .

Finally, we show that $p_{0}$ has finite kernel. We have

$$
\begin{aligned}
\operatorname{ker} p_{0} & =\{(0,0, \mu) \in \text { End } L \times \text { End } L \times \text { End } M: \mu f=0\} \\
& \cong \operatorname{Hom}(M / f(L), M) \cong \operatorname{Hom}\left(M / f(L), M_{\text {tors }}\right),
\end{aligned}
$$

where the last isomorphism follows from Lemma 6.2 and the assumption that $M / f(L)$ is finite. Invoking Lemma 6.2 again, it follows that $\operatorname{ker} p_{0}$ is finite, so $p_{0}$ is an isogeny. 
Theorem 7.2. Let $L, M$ be finitely generated $R$-modules. Then for any commensurability $c=(X, f, g): L \rightleftharpoons M$, the correspondence $\mathrm{e}(c)$ : End $L \rightleftharpoons$ End $M$ is a ring commensurability, and the induced correspondence a $(c)$ : Aut $L \rightleftharpoons$ Aut $M$ is a group commensurability.

Proof. The correspondence e $(c)$ is canonically isomorphic to the composition of e $\left(c_{f}\right)^{-1}$ : End $L \rightleftharpoons$ End $X$ with $\mathrm{e}\left(c_{g}\right):$ End $X \rightleftharpoons$ End $M$. The correspondences $\mathrm{e}\left(c_{f}\right)$ and $\mathrm{e}\left(c_{g}\right)$ are commensurabilities by Lemma 7.1 , so $\mathrm{e}(c)$ is a commensurability by Proposition 2.6. The assertion on a $(c)$ follows from Theorem 3.8 by passing to the unit groups.

Theorem 7.3. Let $c: L \rightleftharpoons M, d: M \rightleftharpoons N$ be commensurabilities of $R$ modules. Then:

(a) the ring commensurability $\mathrm{e}(d \circ c)$ : End $L \rightleftharpoons$ End $N$ is equivalent (see Definition 2.7) to the composition of ring commensurabilities $\mathrm{e}(d) \circ \mathrm{e}(c)$, and the group commensurability $\mathrm{a}(d \circ c)$ is equivalent to the composition $\mathrm{a}(d) \circ \mathrm{a}(c)$

(b) we have

$$
\begin{aligned}
& \mathrm{i}(\mathrm{e}(d \circ c))=\mathrm{i}(\mathrm{e}(d)) \mathrm{i}(\mathrm{e}(c)), \\
& \mathrm{i}(\mathrm{a}(d \circ c))=\mathrm{i}(\mathrm{a}(d)) \mathrm{i}(\mathrm{a}(c)) .
\end{aligned}
$$

Proof. (a) Write $c=(X, f, g): L \rightleftharpoons M, d=(Y, h, j): M \rightleftharpoons N$. We claim that there is an isogeny

$$
i: \text { End } c \times_{\operatorname{End} M} \text { End } d \rightarrow \operatorname{End}(d \circ c)
$$

that makes the following diagram of endomorphism rings commute:

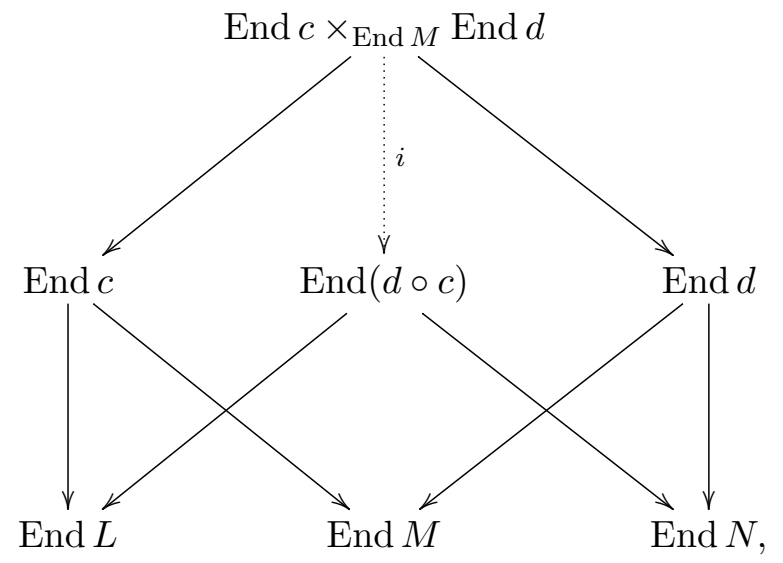

where all unlabelled morphisms are the ones defined in the introduction.

An element of End $c \times_{\operatorname{End} M}$ End $d$ is a pair of triples

$$
\begin{aligned}
& \left((\lambda, \xi, \mu),\left(\mu^{\prime}, v, \nu\right)\right), \\
& \lambda \in \text { End } L, \xi \in \text { End } X, \mu, \mu^{\prime} \in \text { End } M, v \in \text { End } Y, \nu \in \text { End } N,
\end{aligned}
$$

satisfying $\lambda f=f \xi, \mu g=g \xi, \mu^{\prime} h=h v, \nu j=j v$, and the fibre product condition in fact demands that $\mu=\mu^{\prime}$.

An element of $\operatorname{End}(d \circ c)$ is a triple $\left(\lambda^{\prime}, \zeta^{\prime}, \nu^{\prime}\right) \in \operatorname{End} L \times \operatorname{End}\left(X \times \times_{M}\right.$ $Y) \times$ End $N$ satisfying $\lambda^{\prime} f p_{0}=\zeta^{\prime} f p_{0}, \nu^{\prime} j p_{1}=j p_{1} \zeta^{\prime}$, where $p_{0}, p_{1}$ are the 
canonical projection maps from $X \times_{M} Y$ to $X$, respectively $Y$. Define

$$
\begin{aligned}
& i: \quad \text { End } c \times \text { End } M \text { End } d \rightarrow \operatorname{End}(d \circ c) \\
& ((\lambda, \xi, \mu),(\mu, v, \nu)) \mapsto(\lambda,(\xi, v), \nu) .
\end{aligned}
$$

A routine verification, which we leave to the reader, shows that the image of $i$ is indeed contained in $\operatorname{End}(d \circ c)$.

To see that this definition of $i$ makes the above diagram of endomorphism rings commute is also routine, and will also be omitted. It remains to check that $i$ is an isogeny. The correspondence $\mathrm{e}(d) \circ \mathrm{e}(c):$ End $L \rightleftharpoons$ End $N$ consists of End $c \times$ End $M$ End $d$, together with the maps to End $L$ and End $N$. By Theorem 7.2, the correspondences $\mathrm{e}(c)$ and $\mathrm{e}(d)$ are commensurabilities, so by Proposition 2.6 the correspondence $\mathrm{e}(d) \circ \mathrm{e}(c)$ is a commensurability. In particular, the morphism End $c \times_{\operatorname{End} M}$ End $d \rightarrow$ End $L$ is an isogeny. Also, $\operatorname{End}(d \circ c) \rightarrow$ End $L$ is an isogeny by Theorem 7.2. The fact that $i$ is an isogeny therefore follows from Proposition 2.1. This proves our claim.

The isogeny $i$ defines an equivalence between $\mathrm{e}(d \circ c)$ and $\mathrm{e}(d) \circ \mathrm{e}(c)$. This proves part (a) for endomorphism rings. By passing to the unit groups and applying Theorem 3.8 to the isogeny $i$, we also obtain part (a) for automorphism groups.

Part (b) immediately follows from part (a) by Propositions 2.12 and 2.6.

Proposition 7.4. Let $L, M$ be finitely generated $R$-modules, and let $c, d$ : $L \rightleftharpoons M$ be two commensurabilities. If $c$ is equivalent to $d$, then $\mathrm{e}(c)$ is equivalent to $\mathrm{e}(d)$, and $\mathrm{a}(c)$ is equivalent to $\mathrm{a}(d)$.

Proof. Let $c=(X, f, g)$ and $d=(Y, h, j)$. First, assume that an equivalence between $c$ and $d$ is given by an isogeny $p: Y \rightarrow X$, so that we have the following commutative diagram:

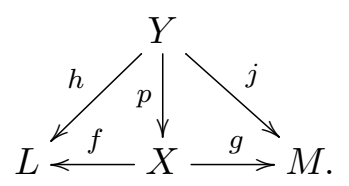

As before, write $c_{f}=(X$,id, $f): X \rightleftharpoons L$, and define $c_{g}, c_{p}$ similarly. Then $d$ is canonically isomorphic to $\left(c_{g} \circ c_{p}\right) \circ\left(c_{p}^{-1} \circ c_{f}^{-1}\right)$. By Theorem 7.3, the commensurability e $(d)$ is equivalent to $\mathrm{e}\left(c_{g}\right) \circ \mathrm{e}\left(c_{p}\right) \circ \mathrm{e}\left(c_{p}\right)^{-1} \circ \mathrm{e}\left(c_{f}^{-1}\right)$. By Proposition 2.13 , the composition e $\left(c_{p}\right) \circ \mathrm{e}\left(c_{p}\right)^{-1}$ is equivalent to (End $\left.X, \mathrm{id}, \mathrm{id}\right):$ End $X \rightleftharpoons$ End $X$. So by Proposition 2.11 the commensurability e $\left(c_{g}\right) \circ \mathrm{e}\left(c_{p}\right) \circ \mathrm{e}\left(c_{p}\right)^{-1} \circ$ $\mathrm{e}\left(c_{f}^{-1}\right)$ is equivalent to $\mathrm{e}\left(c_{g}\right) \circ(\operatorname{End} X, \mathrm{id}, \mathrm{id}) \circ \mathrm{e}\left(c_{f}^{-1}\right)$, which is canonically isomorphic to $\mathrm{e}\left(c_{g}\right) \circ \mathrm{e}\left(c_{f}^{-1}\right)$. Applying Theorem 7.3 again, we find that $\mathrm{e}\left(c_{g}\right) \circ \mathrm{e}\left(c_{f}^{-1}\right)$ is equivalent to $\mathrm{e}\left(c_{g} \circ c_{f}^{-1}\right)$. Finally, $c_{g} \circ c_{f}^{-1}$ is canonically isomorphic to $c$, and the special case of the proposition follows.

Passing to the general case, let $(W, p, q): X \rightleftharpoons Y$ be an equivalence between $c$ and $d$. Since $p$ is an isogeny, $c$ is equivalent to $(W, f p, g p)$, and since $q$ is an isogeny, $d$ is equivalent to $(W, h q, j q)=(W, f p, g p)$. The result therefore follows from the special case we just did.

Let Rng denote the category of rings, and Grp the category of groups. Theorem 7.3 and Proposition 7.4 imply that there is a functor from ${ }_{R} \mathbf{M o d}$ com 
to $\mathbf{R n g}_{\text {com }}$ that takes an $R$-module $L$ to the ring End $L$, and an equivalence class of $R$-module commensurabilities, represented by a commensurability $c$, to the equivalence class of ring commensurabilities represented by e $(c)$. Further, Theorem 3.8 shows that we have the functors ${ }^{+}$and ${ }^{\times}$from $\mathbf{R n g}_{\text {com }}$ to Grp $_{\text {com }}$ which take a ring to the additive, respectively multiplicative group of the ring. Finally, Propositions 2.12 and 2.6 imply that we have the functor i from $\mathbf{G r p}_{\text {com }}$ to the group $\mathbb{Q}_{>0}$, thought of as a groupoid with one object. To summarise, we have the functors of groupoids

$$
{ }_{R} \mathbf{M o d}_{\mathrm{com}} \stackrel{\text { End }}{\longrightarrow} \mathbf{R n g}_{\mathrm{com}} \underset{\times}{\stackrel{+}{\longrightarrow}} \operatorname{Grp}_{\mathrm{com}} \stackrel{\mathrm{i}}{\longrightarrow} \mathbb{Q}>0 .
$$

Let $L$ be a finitely generated $R$-module, and let $V$ denote the $A$-module $Q \otimes_{Z} L$. The isomorphism of Corollary 6.9 and the functors (7.5) then induce group homomorphisms

$$
\begin{aligned}
\operatorname{Aut}_{A} V \cong G_{L} & \rightarrow \mathbb{Q}>0, \\
c & \mapsto \mathrm{i}(\mathrm{e}(c)) \text { and } \\
c & \mapsto \mathrm{i}(\mathrm{a}(c)) .
\end{aligned}
$$

Lemma 7.7. Let $L$ be a finitely generated $R$-module, write $\bar{L}=L / L_{\mathrm{tors}}$, and let $f: L \rightarrow \bar{L}$ denote the quotient map. Then the isomorphism in ${ }_{R} \mathbf{M o d}_{\mathrm{com}}$ given by the commensurability ( $L, \mathrm{id}, f): L \rightleftharpoons \bar{L}$ induces an isomorphism $G_{L} \rightarrow G_{\bar{L}}$ that commutes with the maps $G_{L} \rightarrow \mathbb{Q}_{>0}$ and $G_{\bar{L}} \rightarrow \mathbb{Q}_{>0}$ defined in (7.6).

Proof. Let $t$ denote the commensurability $L \stackrel{\text { id }}{\leftarrow} L \rightarrow \bar{L}$. Then the isomorphism $G_{L} \rightarrow G_{\bar{L}}$ is given by composition on the right with $t$ and on the left with $t^{-1}$. It follows from Theorem 7.3, Proposition 2.6 and Proposition 2.12 that this isomorphism commutes with the maps (7.6).

Proposition 7.8. Let $L$ be a finitely generated $R$-module, and denote the $A$ module $Q \otimes_{Z} L$ by $V$. Let $\alpha$ be an element of $\mathrm{Z}\left(\operatorname{End}_{A} V\right)^{\times} \subset \operatorname{Aut}_{A} V \cong G_{L}$. Then its image in $\mathbf{R n g}_{\text {com }}$ under the first functor of (7.5) is the identity morphism on End $L$.

Proof. By Lemma 7.7, we may assume that $L$ is $Z$-torsion free. Thus, $L$ injects into $V=Q \otimes_{Z} L$. For any sub- $R$-module $U$ of $V$, write $E_{U}=\{\phi \in$ $\left.\operatorname{End}_{A} V: \phi U \subset U\right\}$. Then the injection $L \longmapsto V$ induces a map $\operatorname{End}_{R} L \rightarrow$ $\operatorname{End}_{A} V$, which is injective and whose image is exactly $E_{L}$.

Let $\alpha \in \operatorname{Aut}_{A} V$ be arbitrary. Then the isomorphism $\operatorname{Aut}_{A} V \cong G_{L}$ identifies $\alpha$ with the equivalence class of commensurabilities represented by $c=\left(L \cap \alpha^{-1} L, i, \alpha\right): L \rightleftharpoons L$, where $i: L \cap \alpha^{-1} L \rightarrow L$ is the inclusion map. We have

$$
\begin{aligned}
\text { End } c= & \left\{\left(\lambda_{0}, \lambda_{1}\right) \in \operatorname{End}_{A} V \times \operatorname{End}_{A} V:\right. \\
& \left.\lambda_{0} \in E_{L} \cap E_{\alpha^{-1} L}, \lambda_{1} \in E_{\alpha L} \cap E_{L}, \lambda_{0}=\alpha^{-1} \lambda_{1} \alpha\right\} .
\end{aligned}
$$

The commensurability e $(c)$ is then of the form (End $\left.c, p_{0}, p_{1}\right)$ : End $L \rightleftharpoons$ End $L$, where $p_{0}:\left(\lambda_{0}, \lambda_{1}\right) \mapsto \lambda_{0}$, and $p_{1}:\left(\lambda_{0}, \lambda_{1}\right) \mapsto \lambda_{1}=\alpha \lambda_{0} \alpha^{-1}$.

It follows that if $\alpha$ is an element of $\mathrm{Z}\left(\operatorname{End}_{A} V\right)^{\times}$, then $p_{0}$ and $p_{1}$ are equal. In this case, the commensurability (End $c$, id, $\left.p_{0}\right):$ End $c \rightleftharpoons$ End $L$ defines an equivalence between $\mathrm{e}(c)$ and (End $L, \mathrm{id}, \mathrm{id})$ : End $L \rightleftharpoons$ End $L$, the identity morphism on End $L$ in $\mathbf{R n g}_{\text {com }}$. 
The following result is an immediate consequence of Proposition 7.8.

Corollary 7.9. The two group homomorphisms Aut $_{A} V \rightarrow \mathbb{Q}_{>0}$ of (7.6) factor through Aut $_{A} V / \mathrm{Z}\left(\operatorname{End}_{A} V\right)^{\times}$.

Remark 7.10. The computation in the proof of Proposition 7.8 shows that the group homomorphism i $\circ \mathrm{e}: \operatorname{Aut}_{A} V \rightarrow \mathbb{Q}_{>0}$ is given by

$$
\alpha \mapsto \frac{\left(E_{L}: E_{\alpha L} \cap E_{L}\right)}{\left(E_{L}: E_{L} \cap E_{\alpha^{-1} L}\right)},
$$

and analogously for i $\circ$ a.

\section{The Case of Semisimple algebras}

In this section, we prove our main results. We begin with Theorem 1.2. We recall the statement.

Theorem 8.1. Let $Z$ be an infinite domain such that for all non-zero $m \in Z$ the ring $Z / m Z$ is finite, let $Q$ be the field of fractions of $Z$, let $A$ be a semisimple $Q$-algebra of finite vector space dimension over $Q$, let $R \subset A$ be a sub-Z-algebra with $Q \cdot R=A$, and let $L, M$ be finitely generated $R$-modules. Then:

(a) there is an $R$-module commensurability $L \rightleftharpoons M$ if and only if the $A$ modules $Q \otimes_{Z} L$ and $Q \otimes_{Z} M$ are isomorphic;

(b) if $c: L \rightleftharpoons M$ is an $R$-module commensurability, then $\mathrm{e}(c)$ : End $L \rightleftharpoons$ End $M$ is a ring commensurability, and a $(c)$ : Aut $L \rightleftharpoons$ Aut $M$ is a group commensurability;

(c) if $c, c^{\prime}: L \rightleftharpoons M$ are $R$-module commensurabilities, then one has

$$
\mathrm{i}(\mathrm{e}(c))=\mathrm{i}\left(\mathrm{e}\left(c^{\prime}\right)\right), \quad \mathrm{i}(\mathrm{a}(c))=\mathrm{i}\left(\mathrm{a}\left(c^{\prime}\right)\right) .
$$

Proof. By Theorem 4.2, the ring $R$ is left-noetherian, so the assumptions of Notation 6.1 are satisfied. Parts (a) and (b) of the theorem therefore follow from Theorems 6.3 and 7.2 , respectively.

We now prove part (c). Let $c, c^{\prime}: L \rightleftharpoons M$ be $R$-module commensurabilities. By Theorem 7.3, the assertion of part (c) is equivalent to the statement that

$$
\mathrm{i}\left(\mathrm{e}\left(c^{-1} \circ c^{\prime}\right)\right)=\mathrm{i}\left(\mathrm{a}\left(c^{-1} \circ c^{\prime}\right)\right)=1 .
$$

So we may, without loss of generality, assume that $L=M$, and it suffices to show that the homomorphisms

$$
\text { i } \circ \text { e, i } \circ \text { a: } \operatorname{Aut}_{A} V \cong G_{L} \rightarrow \mathbb{Q}_{>0}
$$

defined in (7.6) are trivial. Here $V$ denotes the $A$-module $Q \otimes_{Z} L$.

Let $B$ denote the $Q$-algebra $\operatorname{End}_{A} V$, so that $G_{L}=B^{\times}$. Since $\mathbb{Q}_{>0}$ is abelian, both homomorphisms i o e and i o a factor through $B^{\times} /\left[B^{\times}, B^{\times}\right]$. By Corollary 7.9 , they also factor through $B^{\times} / \mathrm{Z}(B)^{\times}$. Since $A$ is a semisimple ring, and since $V$ is a finitely generated $A$-module, it follows that $V$ is a finite direct sum of simple modules, so by Schur's lemma $B$ is a direct product of matrix rings over division rings, and in particular a semisimple ring. By Theorem 5.6, the quotient $B^{\times} /\left(\mathrm{Z}(B)^{\times}\left[B^{\times}, B^{\times}\right]\right)$is an abelian group of finite exponent. Since $\mathbb{Q}_{>0}$ is torsion-free, any homomorphism $B^{\times} /\left(\mathrm{Z}(B)^{\times}\left[B^{\times}, B^{\times}\right]\right) \rightarrow \mathbb{Q}_{>0}$ must be trivial. 
Example 8.2. The following example demonstrates that if we replace the semisimplicity assumption on $A$ by the condition that $R$ be left-noetherian, then the conclusion of Theorem 1.2(c) need no longer hold.

Let $R=\left(\begin{array}{c}\mathbb{Z} \\ 0\end{array}\right)$, and $A=\mathbb{Q} \otimes_{\mathbb{Z}} R$. Let $L$ be a free $R$-module of rank 1 , set $V=\mathbb{Q} \otimes_{\mathbb{Z}} L$, and $B=\operatorname{End}_{A} V$. We have End $L \cong R^{\text {opp }}$, and similarly

$$
B^{\times} \cong\left(A^{\text {opp }}\right)^{\times} \cong\left(\begin{array}{cc}
\mathbb{Q}^{\times} & 0 \\
\mathbb{Q} & \mathbb{Q}^{\times}
\end{array}\right) \text {. }
$$

Recall from equation (7.6), that i o e defines a group homomorphism from $B^{\times}$to $\mathbb{Q}>0$, which factors through $B^{\times} /\left(\mathrm{Z}(B)^{\times} \cdot\left[B^{\times}, B^{\times}\right]\right)$. The map $\left(\begin{array}{ll}a & 0 \\ b & c\end{array}\right) \mapsto$ $c / a$ defines an isomorphism of this quotient with $\mathbb{Q}^{\times}$. For $\alpha=\left(\begin{array}{ll}1 & 0 \\ 0 & c\end{array}\right)$, one easily computes, using Remark 7.10, that $\mathrm{i}(\mathrm{e}(\alpha))=\mathrm{i}(\mathrm{a}(\alpha))=|c|$. It follows that both ioe and i o a map $\left(\begin{array}{ll}a & 0 \\ b & c\end{array}\right)$ to $|c / a|$, and are therefore far from trivial.

We now deduce Theorem 1.1.

Theorem 8.3. Let $G$ be a finite group, let $V$ be a finitely generated $\mathbb{Q}[G]$ module, and put $\mathcal{S}=\left\{L: L\right.$ is a finitely generated $\mathbb{Z}[G]$-module with $\mathbb{Q} \otimes_{\mathbb{Z}}$ $L \cong V$ as $\mathbb{Q}[G]$-modules $\}$. Then there exists a unique function ia: $\mathcal{S} \times \mathcal{S} \rightarrow$ $\mathbb{Q}>0$ such that

(a) if $L, L^{\prime}, M, M^{\prime} \in \mathcal{S}$ and $L \cong L^{\prime}, M \cong M^{\prime}$, then $\operatorname{ia}(L, M)=\mathrm{ia}\left(L^{\prime}, M^{\prime}\right)$;

(b) if $L, M, N \in \mathcal{S}$, then $\operatorname{ia}(L, M) \cdot \mathrm{ia}(M, N)=\mathrm{ia}(L, N)$;

(c) if $M \in \mathcal{S}$, and $L \subset M$ is a submodule of finite index, then with $H=$ $\{\sigma \in$ Aut $M: \sigma L=L\}$ and $\rho: H \rightarrow$ Aut $L$ mapping $\sigma \in H$ to $\sigma \mid L$, one has

$$
\operatorname{ia}(L, M)=\frac{(\operatorname{Aut} M: H) \cdot \# \operatorname{ker} \rho}{(\operatorname{Aut} L: \rho H)} .
$$

Proof. Existence immediately follows from Theorem 8.1: for $L, M \in \mathcal{S}$, we may define ia $(L, M)=\mathrm{i}(\mathrm{a}(c))$ for any commensurability $c: L \rightleftharpoons M$. In particular, property (c) follows by taking the commensurability $c=$ $(L, \mathrm{id}, i): L \rightleftharpoons M$, where $i: L \rightarrow M$ is the inclusion map, and noting that in this case, a $(c)$ is the commensurability Aut $L \stackrel{\rho}{\leftarrow} H \longmapsto$ Aut $M$.

To show uniqueness, observe that the conditions of the theorem imply that the function ia, if it exists, is uniquely determined by its values on $\mathbb{Z}$-free modules. Indeed, if $m_{1}$ and $m_{2}$ are the exponents of the $\mathbb{Z}$-torsion submodule of $L$, respectively of $M$, then condition (b) requires that

$$
\operatorname{ia}\left(m_{1} L, L\right) \operatorname{ia}(L, M)=\operatorname{ia}\left(m_{1} L, m_{2} M\right) \operatorname{ia}\left(m_{2} M, M\right) .
$$

Condition (c) determines the values of ia $\left(m_{1} L, L\right)$ and ia $\left(m_{2} M, M\right)$, so ia $(L, M)$ is determined by ia $\left(m_{1} L, m_{2} M\right)$. Clearly, the modules $m_{1} L$ and $m_{2} M$ are both $\mathbb{Z}$-free.

But if $L, M$ are $\mathbb{Z}$-free, and $\mathbb{Q} \otimes_{\mathbb{Z}} L \cong_{\mathbb{Q}[G]} \mathbb{Q} \otimes_{\mathbb{Z}} M$, then there exists an embedding $L \longmapsto M$ with finite index, in which case ia $(L, M)$ is determined by conditions (a) and (c).

The first interesting case of Theorem 1.1 is already when $G$ is the trivial group, so that finitely generated $\mathbb{Z}[G]$-modules are just finitely generated abelian groups.

Proposition 8.4. Let $L, M$ be finitely generated abelian groups. Then: 
(a) there exists a commensurability $L \rightleftharpoons M$ if and only if $L$ and $M$ have the same rank;

(b) if $L \cong \mathbb{Z}^{n} \oplus L_{0}$ and $M \cong \mathbb{Z}^{n} \oplus M_{0}$, where $L_{0}$ and $M_{0}$ are finite abelian groups, then

$$
\operatorname{ia}(L, M)=\frac{\left(\# M_{0}\right)^{n} \cdot \# \text { Aut } M_{0}}{\left(\# L_{0}\right)^{n} \cdot \# \operatorname{Aut} L_{0}} .
$$

Proof. Part (a) immediately follows from Theorem 1.2(a).

We now prove part (b). First we compute ia $\left(\mathbb{Z}^{n}, L\right)$. The split exact sequence

$$
0 \rightarrow L_{0} \rightarrow L \stackrel{f}{\rightarrow} \mathbb{Z}^{n} \rightarrow 0
$$

induces a surjective map

$$
\text { Aut } L \rightarrow \text { Aut } L_{0} \times \text { Aut } \mathbb{Z}^{n} \text {, }
$$

whose kernel is easily seen to be canonically isomorphic to $\operatorname{Hom}\left(\mathbb{Z}^{n}, L_{0}\right)$. It follows that if $c$ is the commensurability $(L, f, \mathrm{id}): \mathbb{Z}^{n} \rightleftharpoons L$, then the map Aut $c \rightarrow$ Aut $L$ is an isomorphism, while the map Aut $c \rightarrow$ Aut $\mathbb{Z}^{n}$ is onto, with kernel of cardinality \# $\operatorname{Hom}\left(\mathbb{Z}^{n}, L_{0}\right) \cdot \#$ Aut $L_{0}=\left(\# L_{0}\right)^{n} \cdot \#$ Aut $L_{0}$. Hence ia $\left(\mathbb{Z}^{n}, L\right)=\mathrm{i}(\mathrm{a}(c))=\left(\# L_{0}\right)^{n} \cdot \#$ Aut $L_{0}$.

It follows from the above computation that

$$
\begin{aligned}
\operatorname{ia}(L, M) & =\frac{\mathrm{ia}\left(\mathbb{Z}^{n}, M\right)}{\mathrm{ia}\left(\mathbb{Z}^{n}, L\right)} \\
& =\frac{\left(\# M_{0}\right)^{n} \cdot \# \text { Aut } M_{0}}{\left(\# L_{0}\right)^{n} \cdot \# \operatorname{Aut} L_{0}},
\end{aligned}
$$

as claimed.

\section{REFERENCES}

[1] E. Artin, Geometric Algebra, Interscience Publishers Inc., New York (1957).

[2] N. Bourbaki, Éléments de mathématique, Algèbre, Chapitre 8, Modules et anneaux semi-simples, Appendice 2.

[3] H. Cohen and H. W. Lenstra, Jr., Heuristics on class groups of number fields, Number theory, Noordwijkerhout 1983, Lecture Notes in Math. 1068, Springer, Berlin (1984), $33-62$.

[4] H. Cohen and J. Martinet, Étude heuristique des groupes de classes des corps de nombres, J. Reine Angew. Math. 404 (1990), 39-76.

[5] I. S. Cohen, Commutative rings with restricted minimum condition, Duke Math. J. 17 (1950), 27-42.

[6] C. W. Curtis and I. Reiner, Methods of Representation Theory with Applications to Finite Groups and Orders, Vol. 1, John Wiley \& Sons, New York (1981).

[7] P. Gabriel and M. Zisman, Calculus of Fractions and Homotopy Theory, Ergebnisse der Mathematik und ihrer Grenzgebiete 35, Springer, New York (1967).

[8] T. Y. Lam, A First Course in Noncommutative Rings, GTM 131, Springer, New York (2001).

[9] S. Lang, Algebra, GTM 211, Springer, New York (2002).

[10] H. W. Lenstra, A normal basis theorem for infinite Galois extensions, Indag. Math. 88 (1985), 221-228.

[11] J. Lewin, Subrings of finite index in finitely generated rings, J. Algebra 5 (1967), 84-88.

[12] The Stacks Project Authors, Stacks Project, http://stacks.math.columbia.edu (2016).

[13] J. H. M. Wedderburn, On division algebras, Trans. Amer. Math. Soc. 22 (1921), 129-135. 
Mathematics Institute, Zeeman Bullding, University of Warwick, Coventry CV4 7AL, UK

Mathematisch Institudt, Universiteit Leiden, Postbus 9512, 2300 RA Leiden, The NetherLands 